\title{
LipoGlo: A sensitive and specific reporter of atherogenic lipoproteins
}

\author{
James H. Thierer ${ }^{1,2}$, Stephen C. Ekker ${ }^{3}$ and Steven A. Farber ${ }^{1,2 *}$
}

${ }^{1}$ Carnegie Institution for Science Department of Embryology, Baltimore, MD 21218

\section{ABSTRACT:}

Apolipoprotein-B (APOB) is the structural component of atherogenic lipoproteins, lipid-rich

16 particles that drive atherosclerosis by accumulating in the vascular wall. As atherosclerotic

17 cardiovascular disease is the leading cause of death worldwide, there is an urgent need to develop new

18 strategies to prevent lipoproteins from causing vascular damage. Here we report the LipoGlo system,

19 which uses a luciferase enzyme (NanoLuc) fused to ApoB to monitor several key determinants of

20 lipoprotein atherogenicity including particle abundance, size, and localization. Using LipoGlo, we are

21 able to comprehensively characterize the lipoprotein profile of individual larval zebrafish and collect the

22 first images of atherogenic lipoprotein localization in an intact organism. We discover multiple

23 unexpected extravascular lipoprotein localization patterns, as well as identify pla2g12b as a potent

24 regulator of lipoprotein size. ApoB-fusion proteins thus represent a uniquely sensitive and specific

25 approach to study atherogenic lipoproteins and their genetic and small molecule modifiers.

\section{KEYWORDS:}

28 Lipoprotein, Apolipoprotein-B, ApoB, atherosclerosis, cardiovascular disease, zebrafish, pla2g12b 


\section{INTRODUCTION:}

ApoB-containing lipoproteins (ABCLs) are the etiological agents of atherosclerotic

33 cardiovascular disease [1], which is the leading cause of mortality worldwide [2]. ABCLs serve to shuttle

34 lipids throughout the circulation, but occasionally cross the vascular endothelium to form lipid-rich

35 deposits within the vascular wall that develop into atherosclerotic plaques [1]. ABCLs are frequently

36 characterized indirectly through measurement of their triglyceride and cholesterol content, and high-risk

37 individuals with elevated lipid levels are prescribed lipid-lowering therapies such as statins [3]. Such

38 drugs effectively reduce cardiovascular disease risk by lowering the levels of cholesterol carried by

39 atherogenic lipoproteins (often called "bad cholesterol").

40 Indirect (lipid-focused) measurements, however, provide very limited information on $A B C L$

41 properties such as particle concentration or size distribution, both of which are key determinants of

42 atherogenic potential. For example, serum Apolipoprotein-B (ApoB) levels directly reflect the

43 concentration of $\mathrm{ABCL}$ particles and show a stronger correlation with cardiovascular disease risk than

44 lipid metrics (including cholesterol) $[4,5]$. The size distribution of lipoprotein particles is also relevant to

45 cardiovascular disease risk, as there are numerous classes of ABCLs that can be differentiated by size

46 and show varying degrees of atherogenicity [6]. Low-density lipoproteins (LDL) are the smallest and

47 most abundant class of ABCLs and are thought to be the primary drivers of atherosclerosis. There is

48 significant size variation within the LDL particle class, and smaller particles are associated with

49 increased atherogenicity [7]. For example, approximately $25 \%$ of the adult population produces

50 unnaturally small LDL particles, and as a result have $\sim 3$-fold higher risk for cardiovascular disease [8].

51 Many of the genetic and environmental factors governing ABCL size and abundance remain

52 undiscovered or poorly characterized [9-11], and even fewer have been successfully targeted

53 pharmaceutically [12-14]. It has proven particularly difficult to identify drugs that modulate ABCL size

54 and abundance because the simplified model systems (such as cultured cells or invertebrate models)

55 typically used in high-throughput drug screening do not recapitulate the complex multi-organ physiology

56 responsible for $A B C L$ homeostasis. While lipoproteins are studied extensively in mammalian models, 
57 these systems are not conducive to high-throughput drug discovery. By contrast, the larval zebrafish

58 model system has proven to be a powerful system for in vivo drug discovery, as it recapitulates all

59 major aspects of vertebrate physiology in a small, transparent, rapidly developing organism. However,

60 no existing assays are sensitive enough to characterize ABCLs in individual larval zebrafish [15-17], as

61 each larvae contains only a few nanoliters of plasma.

62 Here we present the LipoGlo reporter as a remarkably sensitive and tractable new tool to study

63 atherogenic lipoproteins. Modern genome engineering techniques were used to fuse the endogenous

64 ApoB gene in zebrafish with an engineered luciferase reporter (NanoLuc), such that each atherogenic

65 lipoprotein would be tagged with a light-emitting molecule. Using this reporter, we were able to develop

66 several independent assays to characterize distinct aspects of the ABCL profile (summarized in Fig.

67 1a). These include a plate-based assay to measure lipoprotein quantity (LipoGlo-Counting), a gel-

68 based assay to measure lipoprotein size (LipoGlo-Electrophoresis), and chemiluminescent imaging to

69 visualize lipoprotein localization (LipoGlo-Microscopy).

$70 \quad$ We also performed extensive validation of these assays in vivo by showing conserved

71 responses to genetic, pharmacological, and dietary manipulations in living zebrafish (summarized in

72 Fig. 1b). Finally, we leveraged the discovery potential of these assays to identify previously

73 uncharacterized associations between ABCLs and the central nervous system [18], as well as identify

74 the poorly characterized gene pla2g12b[19] as a potent regulator of lipoprotein particle size that is

75 conserved across vertebrates.

76 LipoGlo was developed first in larval zebrafish as this organism is uniquely well-suited for high-

77 throughput genetic and small molecule screening, as well as whole-organism imaging. However,

78 LipoGlo represents a highly generalizable tool that can be expanded to function in essentially any

79 organism with atherogenic lipoproteins, and customized with different reporters depending on the

80 research question. This technique has the potential to transform our understanding of atherogenic

81 lipoprotein biology, which may have important clinical repercussions in the treatment of atherosclerotic

82 cardiovascular disease. 

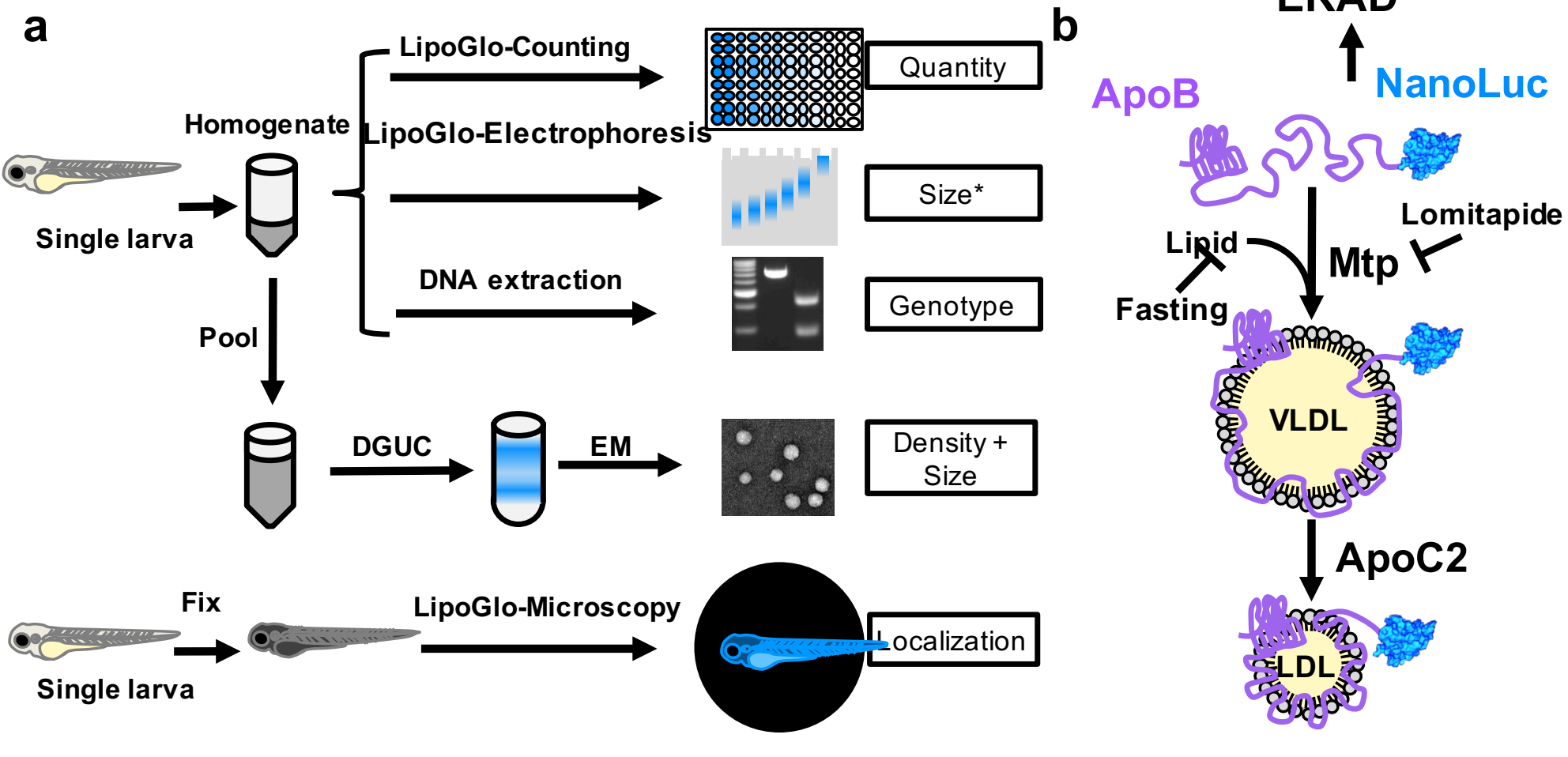

\section{Figure 1:}

Overview of LipoGlo assays and experimental manipulations. (a) Individual larvae carrying the ApoB-NanoLuc reporter are first homogenized in ABCL stabilization buffer. Homogenate can be used for LipoGlo-Counting (a plate-based assay for NanoLuc activity to measure the total number of $A B C L s$ ), LipoGlo-Electrophoresis (a Native-PAGE assay to determine the $A B C L$ size/subclass distribution), and DNA extraction for genotyping. Alternatively, lipoprotein density and size can be determined by density-gradient ultracentrifugation on pooled samples (DGUC) followed by electron microscopy. To determine localization of ABCLs in situ, individual larvae are fixed in 4\% PFA and mounted in low-melt agarose for chemiluminescent imaging (LipoGloMicroscopy). Asterisk indicates that electrophoretic mobility is an indirect measure of particle size. (b) ApoB protein fused to NanoLuc is loaded with lipid through the activity of Mtp to form VLDL particles. In the absence of lipidation, the protein will be rapidly degraded by ERAD. VLDL is lipolyzed by serum lipases that use Apoc2 as an obligate cofactor to produce smaller lipoprotein classes such as LDL. Here we investigate the effects of (i) genetic manipulations (mutations in mtp and apoC2), (ii) dietary variation (fasting and feeding), and (iii) pharmacological treatment (inhibition of Mtp with lomitapide) on various aspects of the $A B C L$ profile. 
RESULTS:

TALEN-mediated genome engineering enables creation of the LipoGlo reporter

$A p o B$ is an ideal scaffold for creating a reporter of $A B C L s$. It is both an obligate structural component present in single copy on each lipoprotein particle [20], and is rapidly degraded when not associated with an ABCL via endoplasmic-reticulum-associated protein degradation (ERAD) [21] (Fig. 1b). In mammals there is a single $A P O B$ gene that can be post-transcriptionally edited into two isoforms: the full-length $A P O B-100$ expressed primarily in the liver, and the truncated $A P O B-48$ isoform expressed in the intestine $[22,23]$. Although the zebrafish genome contains 3 paralogs of

$94 A P O B$, a single paralog (apoBb.1) is the clearly dominant isoform, accounting for approximately $95 \%$

95 of the $A p o B$ mRNA and protein in larval zebrafish [24]. Known functional elements of ApoB are well conserved in zebrafish, including both the microsomal triglyceride transfer protein (MTP) interacting

97 [25] and LDL-receptor binding [26] domains (Supplementary Fig. 1a). However, the APOB-48 editing 98 site required for production of the truncated (intestine-specific) version of APOB [23] appears to be 99 completely absent in zebrafish (Supplementary Fig. 1b). This creates the opportunity to

100 simultaneously tag both intestine and liver derived ABCLs with a carboxy-terminal fusion to ApoBb.1 101 in zebrafish.

NanoLuc is an optimized luciferase reporter that generates a quantitative chemiluminescent

103 signal through cleavage of its substrate molecule, furimazine [27]. This reporter is remarkably bright

104 ( 100 times brighter than firefly luciferase), small (19.1 kDa), stable, and provides robust signal to

105 noise ratios that enable accurate detection even at femtomolar concentrations [27]. The NanoLuc

106 coding sequence was introduced as a carboxy-terminal fusion to the endogenous ApoBb.1 gene in

107 zebrafish through homology directed repair of a double-stranded break [28]. Capped mRNA

108 encoding a TALEN pair targeting the ApoBb.1 stop codon was co-injected with a donor DNA

109 construct to induce homology-directed repair. The donor construct contains the NanoLuc coding

110 sequence flanked on either side by several hundred base pairs of sequence homologous to the

111 genomic sequence upstream and downstream of the ApoBb.1 stop codon (Supplementary Fig. 2).

112 Injected embryos were raised to adulthood and their progeny were screened for NanoLuc activity 
113 and subsequently for error-free integration at the target locus. The resulting tagged lipoproteins are

114 quantified using the Nano-Glo assay (Promega Corp., N1110), which led us to name this system

115 LipoGlo.

116 Fish homozygous for the LipoGlo reporter are healthy, fertile, and do not display any

117 abnormal morphological or behavioral phenotypes. Additionally, larvae homozygous for the LipoGlo

118 reporter show a two-fold increase in LipoGlo signal relative to their heterozygous siblings

119 (Supplementary Fig. 2c). Together, these data suggest that the LipoGlo reporter does not disrupt

120 normal production, secretion, and turnover of lipoprotein particles.

\section{LipoGlo-Counting reveals changes in ABCL abundance}

124 The LipoGlo-Counting method uses a 96-well plate based assay to detect NanoLuc activity

125 and quantify $A B C L$ abundance. In order to validate the LipoGlo reporter and evaluate the degree of

126 similarity between zebrafish and mammalian lipoprotein homeostasis, the lipoprotein profile was

127 assayed across development, as well as in response to genetic, pharmacological, and dietary

128 manipulation (Fig. 1b). Individual larvae carrying the LipoGlo reporter are homogenized in a

129 standard volume of $\mathrm{ABCL}$ stabilization buffer $(100 \mu \mathrm{L})$ using either a pellet pestle for low throughput

130 sample processing (Fisher scientific, 12-141-363), or a microplate horn sonicator for processing of

13196 samples simultaneously in plate format (QSonica, 431MPX). The ABCL stabilization buffer

132 contains protease inhibitors, $\mathrm{pH}$ buffers, and cryoprotectant to ensure sample stability during

133 processing and storage. A portion of the homogenate $(40 \mu \mathrm{L})$ is mixed with an equal volume of

134 Nano-Glo assay buffer and quantified in a plate reader. The remaining homogenate is either stored

135 frozen for later use, or used for additional assays (Fig. 1a).

$136 \quad A B C L$ levels were measured throughout development from $1-6$ days post-fertilization (dpf)

137 using zebrafish carrying the LipoGlo reporter in the wild-type (WT) genetic background (Fig. 2a).

138 During this window of development, embryos are in the lecithotropic (yolk-metabolizing) stage [29].

139 All nutrients required for development are provided by the maternally deposited yolk, until the yolk

140 becomes depleted between 5 and $6 \mathrm{dpf}$ and the larvae begin to rely on exogenous food. Yolk lipid is 

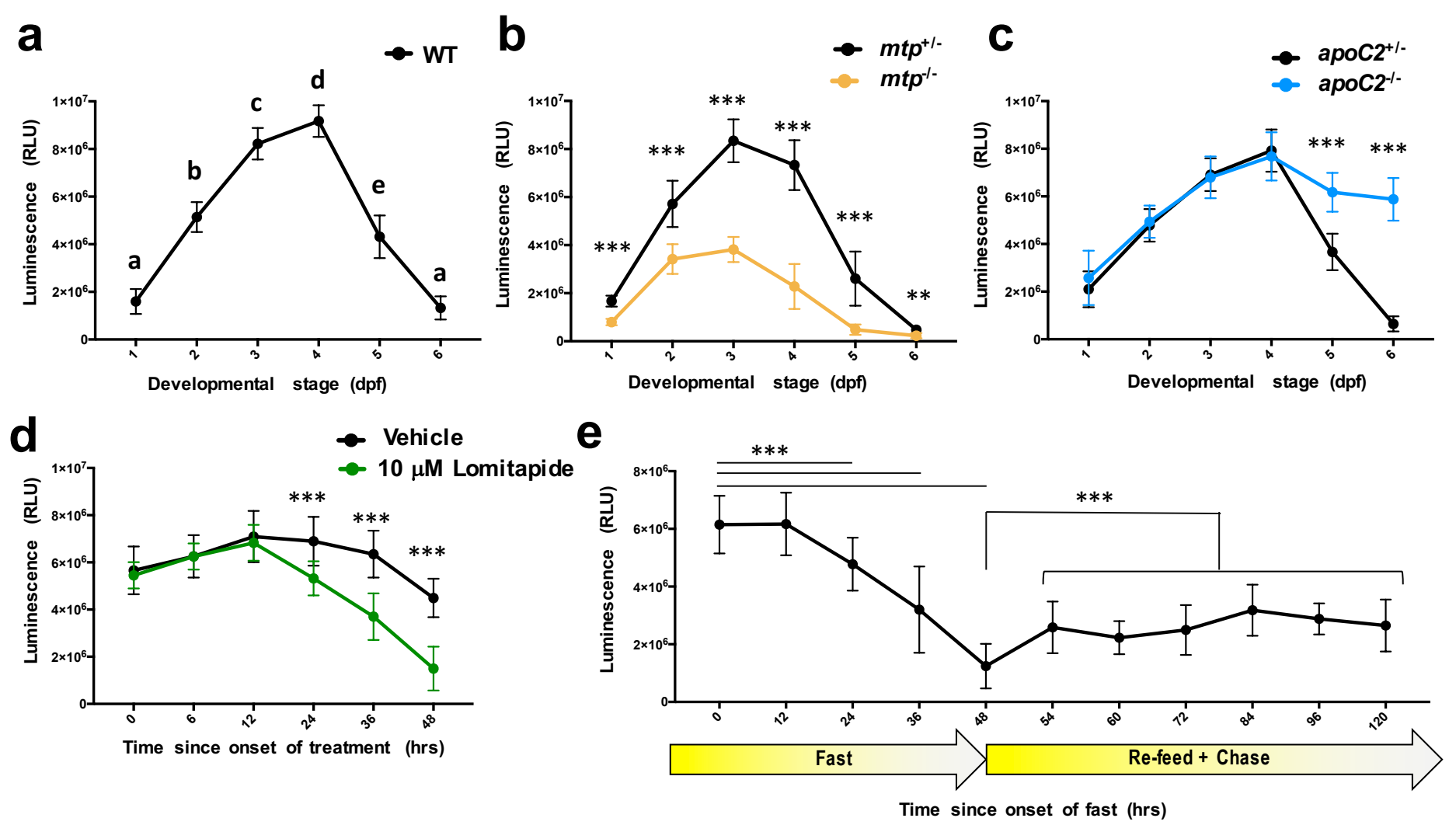

Figure 2:

LipoGlo-Counting reveals conserved $\mathrm{ABCL}$ responses to genetic, dietary, and pharmacological stimuli. (a) LipoGlo signal throughout WT larval zebrafish development (1-6 dpf). Time points designated with different letters are statistically significantly different ( $n=24$, ANOVA $p<0.0001$, Tukey's HSD $p<.0001$ ). (b) Comparison of LipoGlo signal between $m t p^{-/-}$mutants (defective in lipoprotein synthesis) and $m t p^{+-}$siblings during larval development ( $\approx \approx 16$, Two-way robust ANOVA p<0.0001 for genotype and stage, Games-Howell p<.001). (c) Comparison of LipoGlo signal between apoC2/- mutants (defective in lipoprotein breakdown) and $a p o C 2^{+/-}$siblings during larval development ( $\approx \approx 12$, Two-way robust ANOVA $p<0.0001$ for genotype

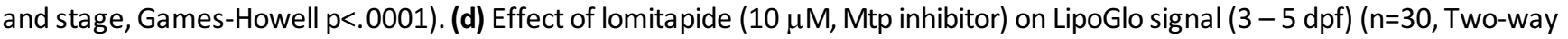
robust ANOVA $p<0.0001$ for treatment and time, Games-Howell $\mathrm{p<.0001).} \mathrm{(e)} \mathrm{LipoGlo} \mathrm{levels} \mathrm{were} \mathrm{measured} \mathrm{over} \mathrm{time}$ throughout a fast, re-feed, and chase period. Larvae were fed a standard diet ad libitum from 5 to $10 \mathrm{dpf}$, and then were deprived of food for 48 hours (fast period). Larvae were then fed a high-fat ( $5 \%$ egg yolk) diet for 6 hours, transferred to fresh media, and assayed at various time points for the 72 hours following the onset of feeding (48-120 hours) ( $n=30$, Welch's ANOVA $p<0.0001$, Games-Howell $p<.0001)$. Results represent pooled data from three independent experiments, " $n$ " denotes number of samples per data point. 
141 packaged into ABCLs by the yolk syncytial layer (YSL), a specialized embryonic organ that

142 expresses many genes involved in $\mathrm{ABCL}$ production including ApoBb.1 [24]. Accordingly, ABCL

143 levels are quite low early in development, but increase between $1-3 \mathrm{dpf}$ as more yolk lipid is

144 packaged into ABCLs (Fig. 2a). As the larvae are not provided with food, $A B C L$ levels drop later in

145 development as rates of lipoprotein metabolism and turnover exceed rates of production following

146 yolk depletion.

$147 \quad$ LipoGlo reporter fish were then crossed with fish harboring mutations in essential

148 components of the ABCL production and breakdown pathways. Microsomal Triglyceride Transfer

149 Protein (Mtp) is responsible for loading nascent ApoB with lipid to form ABCLs [30], and

150 Apolipoprotein-C2 (ApoC2) is a cofactor for lipoprotein lipolysis [31] (outlined in Fig. 1b). As

151 expected, $m t p^{-/}$mutants [32] exhibit profound defects in ABCL production detectable from the

152 earliest stages of development (Fig. 2b). By contrast, apoC2-- mutants [15] produce lipoproteins

153 normally but show significantly reduced levels of particle breakdown and turnover compared to

154 sibling controls (Fig. 2c).

155 To probe the effects of transient Mtp inhibition on larval lipoprotein homeostasis, larvae were

156 exposed to lomitapide. Lomitapide is a pharmaceutical inhibitor of Mtp used to treat familial

157 hypercholesterolemia in humans [33]. Larvae were treated with $10 \mu \mathrm{M}$ lomitapide or vehicle control

158 for $48 \mathrm{~h}$ (3-5 dpf), and treated larvae showed a more rapid decline in NanoLuc levels than vehicle-

159 treated controls. This observation is consistent with lomitapide inhibiting ABCL production and

160 leading to an accelerated decline of ApoB-NanoLuc levels (Fig. 2d).

161 To test the effect of food intake on ABCL levels, larvae were subjected to a fasting and re -

162 feeding experimental paradigm. Larvae were fed a standard diet (Gemma 75, Skretting USA) for 5

163 days (from 5-10 dpf) to adapt to food intake and reach a physiologically relevant baseline level of

164 ABCLs. Following the initial feeding period, larvae were fasted for $48 \mathrm{~h}$ (sampled every $12 \mathrm{~h}$ ), re-fed

165 with a high-fat meal of 5\% egg-yolk [34], and sampled at various time points after the meal (the

166 chase period). ApoB-NanoLuc levels were stable for the first $12 \mathrm{~h}$ of the fast, but declined rapidly for

167 the duration of the fasting period (Fig. $2 e, 0-48$ h). Following the high fat meal (6 h of feeding from

168 time point $48 \mathrm{~h}$ to time point $54 \mathrm{~h}$ ), there was an immediate increase in ApoB-NanoLuc levels (Fig. 
$1692 \mathrm{e}, 48-120 \mathrm{hrs}$ ). ApoB-NanoLuc levels did not recover to their pre-fasted state following the high-fat

170 meal, but rather remained at an intermediate level for a prolonged period (the duration of the chase

171 period, $72 \mathrm{~h})$.

172

173 Determination of lipoprotein size distribution using LipoGlo-Electrophoresis

174 There are numerous classes of ABCLs, many of which can be differentiated based on

175 particle size [35]. Native polyacrylamide gel electrophoresis (Native-PAGE) has previously been

176 used to separate ABCLs based on size, but requires a relatively large volume of plasma $(25 \mu \mathrm{L})$

177 stained with lipophilic dyes [36]. The LipoGlo-Electrophoresis method subjects crude larval

178 homogenate (containing only nanoliters of plasma) to Native-PAGE to separate lipoproteins,

179 followed by in-gel detection of NanoLuc activity. To analyze the ABCL size distribution over

180 development and in response to genetic, pharmacological, and dietary manipulations, representative

181 frozen aliquots of larval homogenate from a given condition were thawed on ice. A portion of the

182 thawed homogenate $(12 \mu \mathrm{L})$ was mixed with $5 x$ loading dye $(3 \mu \mathrm{L})$ and separated via Native-PAGE

183 (3\% gel for 275 Volt-h). Following separation, the glass front plate was removed to expose the gel

184 surface, and $1 \mathrm{~mL}$ of TBE containing Nano-Glo substrate solution $(2 \mu \mathrm{L})$ was added to the plate and

185 spread evenly using a thin plastic film. The gel was then imaged using the Odyssey Fc

186 chemiluminescent detection system (LI-COR Biosciences). Together, this protocol is referred to as

187 LipoGlo electrophoresis.

188 Smaller lipoproteins are expected to migrate further into the gel, and larger lipoproteins to 189 show concomitantly less mobility (Fig. 3a). Following electrophoretic separation, ABCLs can be

190 divided into four different classes based on their migration distance. ABCLs that remain within the

191 loading well are classified as the "zero mobility" (ZM) fraction, which should include chylomicrons

192 [37], remnants, aggregates [38], and intracellular ApoB complexed with components of the secretory

193 pathway (such as the ER, golgi, and other secretory vesicles) [39]. Species that do migrate into the

194 gel are classified as either Very Low-Density Lipoproteins (VLDL), Intermediate-Density Lipoproteins

195 (IDL), or Low-Density Lipoproteins (LDL) based on their electrophoretic mobility. Di-I-Labeled

196 fluorescent LDL (L3482, ThermoFisher Scientific) is used as a migration standard to ensure 
a

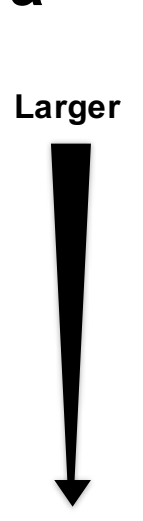

Smaller

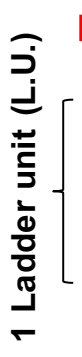

$$
\text { Dil- }
$$

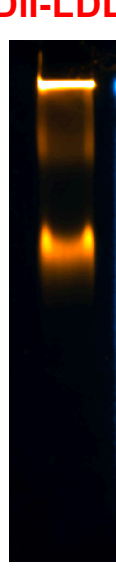

C

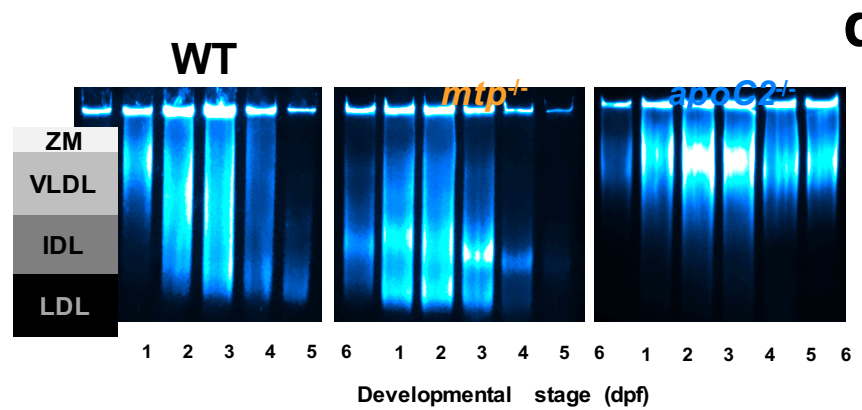
$m t p^{-1 .}$ apoC2-

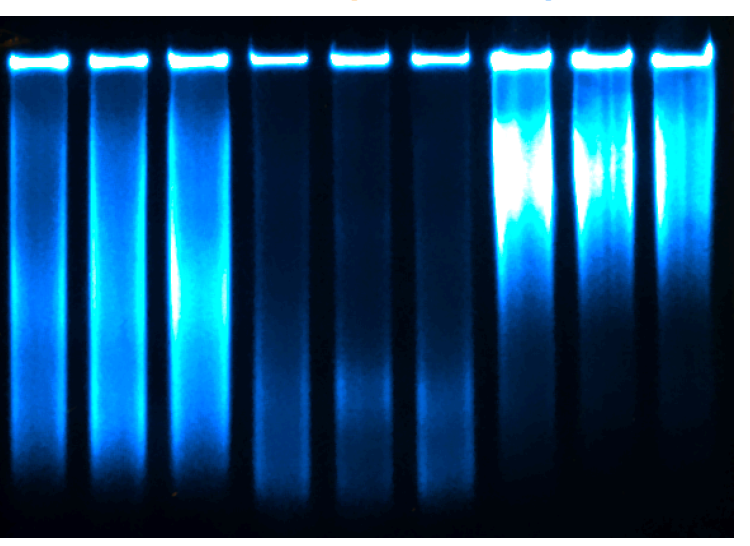

d Developmental stage (dpf)

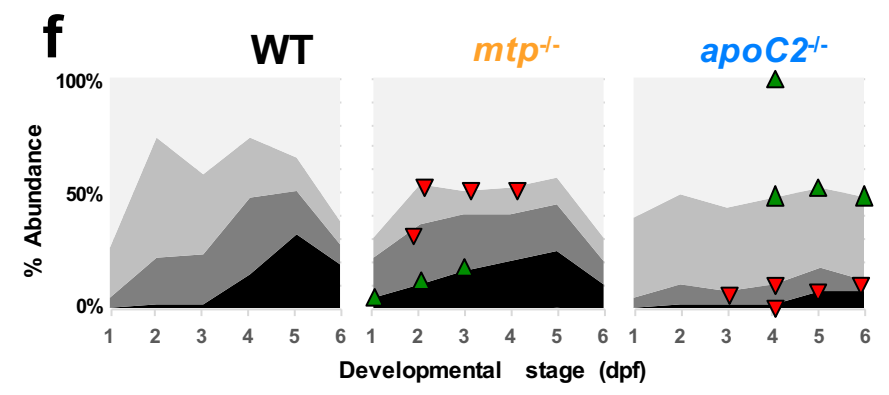

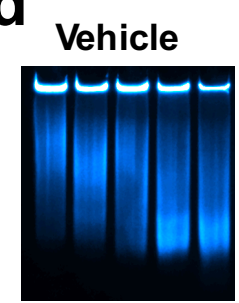

$\begin{array}{lllll}0 & 12 & 24 & 36 & 48\end{array}$ Time since onset of treatment (hrs) b

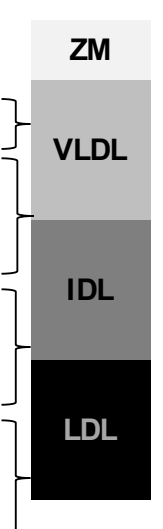

$\mathbf{g}$ Vehicle Lomitapide $\mathbf{h}$ $\nabla \nabla$

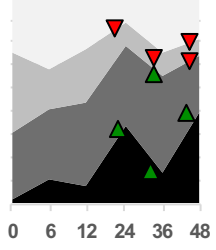

$\begin{array}{llllllllllll}0 & 6 & 12 & 24 & 36 & 48 & 0 & 6 & 12 & 24 & 36 & 48\end{array}$

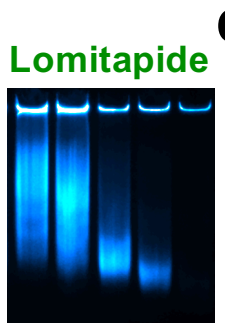

$\begin{array}{llllllllll}0 & 24 & 36 & 48 & 54 & 60 & 72 & 84 & 96 & 120\end{array}$

Time since onset of fast (hrs)
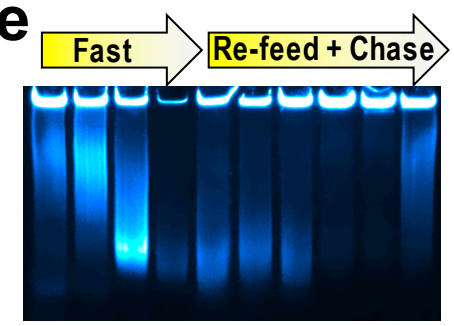

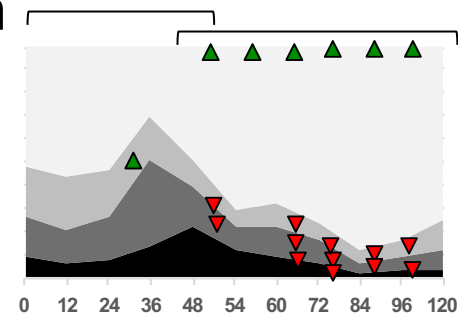

Time since onset of fast (hrs)

Figure 3:

Changes in lipoprotein size distribution revealed through LipoGlo-Electrophoresis. (a) Representative image of the fluorescent Dil-LDL migration standard and LipoGlo emission from WT, $m t^{-/}$, and apoC2 ${ }^{-/}$genotypes (4 dpf). ABCLs are divided into 4 classes based on their mobility, including ZM (zero mobility) and three classes of serum ABCLs (VLDL, IDL, LDL). Image is a composite of chemiluminescent (LipoGlo, blue) and fluorescent (Dil-LDL, orange) exposures. Gel is a representative image from one of the three independent experiments performed. (b) Vertical plot profile generated in ImageJ from gel image displayed in (a), note that the ZM peak has been appended to highlight differences in serum lipoprotein classes. (c-e) Representative gel images (one of three independent experiments shown) and (f-h) pooled LipoGlo-electrophoresis quantification data from larval lysates used in Figure 2. Relative abundance of subclasses is color-coded as shown in (a). Upward-facing arrowheads (green) indicate significant enrichment of that species at that time point compared to controls, and downward-facing arrowheads (red) indicate depletion (f) Subclass abundance at each day of larval development in WT ( $n=9$, Welch's ANOVA p<0.0001 for each subclass over time), $m t p^{-/}(n=9$, Two-way robust ANOVA $p<0.001$ for VLDL and LDL, Games-Howell $p<.01)$, and apoC2 ${ }^{--}$( $n=9$, Two-way robust ANOVA $p<0.01$ for all classes, Games-Howell $p<.005$ ) genetic backgrounds. (g) Subclass abundance from 3-5 $\mathrm{dpf}$ in larvae treated with $10 \mu \mathrm{M}$ lomitapide or vehicle control $(n=9$, Two-way robust ANOVA $p<0.001$ for all classes except IDL, Games-Howell p<.01 ). (h) Subclass abundance from 10-15 dpf in larvae subjected to a fasting and re-feeding paradigm. The first bracket delineates changes relative to time 0 (the onset of the fasting period), and the second bracket delineates changes relative to time point 48 (the onset of the re-feeding period) ( $n=9$, Welch's ANOVA $p<0.0001$ for each subclass over time, Games-Howell p<.01). Supplementary Figure 4 displays standard deviations for panels $\mathrm{f}$-h. Results represent pooled data from three independent experiments, " $n$ " denotes number of samples per data point. 
197 consistent classification of ABCL species between gels, with the migration distance of this species

198 corresponding to one ladder unit (L.U.). Although the Di-I stain significantly reduces electrophoretic

199 mobility of the human LDL and therefore does not align with NanoLuc-labeled LDL (data not shown),

200 this band provides a highly reproducible standard for registration and normalization across gels

201 (Supplementary Fig. 3).

202 In order to define physiologically relevant migration boundaries between ABCL classes,

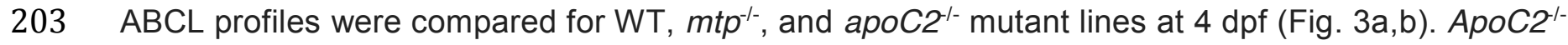

204 mutants are unable to lipolyze VLDL, which allowed us to define the VLDL bin from .3 - 1 ladder

205 units. Conversely, $m t^{-/-}$mutants display a bimodal peak of small LDL-like particles at this stage of

206 development, which was used to define the LDL bin as $1.7-2.4$ ladder units from the origin. Wild-

207 type larvae have a peak of intermediate-sized lipoproteins at this stage, which corresponds to the

208 IDL region from $1-1.7$ ladder units. ABCLs migrating less than 3 ladder units were considered to

209 be in the zero-mobility fraction (ZM) (Fig. 3a).

210 Gel images were transformed into plot profiles in ImageJ for quantification (Fig. 3b). The

211 provided Gel Quantification Template (supplementary file 1) contains instructions and formulas for

212 automatically calculating bin cutoffs for each ABCL class based on the migration of the Di-I standard

213 and quantifying the relative intensity of each bin. To visualize the distribution of ABCL classes over

214 time, each species was color coded with darker colors corresponding to smaller lipoproteins and

215 plotted as an 100\% stacked area chart (Fig. 3f-h). Upward-facing green arrowheads or downward-

216 facing red arrowheads are used to indicate which species show significant enrichment or depletion

217 (respectively) relative to the control group (Fig. 3f-h). Additional plots were generated that present

218 these data grouped by ABCL class (rather than genotype) (Supplementary Fig. 4).

219 Using LipoGlo-Electrophoresis over the course of zebrafish larval development revealed that

220 in the early embryonic stages (1-2 dpf), the wild-type ABCL profile is dominated by VLDL (Fig. 3c,f),

221 which are directly produced by the YSL. By 3 and $4 \mathrm{dpf}$, these VLDL particles have been lipolyzed to

222 generate the smaller IDL and LDL classes. When the maternal yolk has been depleted (5-6 dpf) and

223 in the absence of exogenous food, VLDL production is significantly attenuated as indicated by the

224 enrichment of the small lipolyzed lipoproteins. The ABCL profile dynamics are much more static in 
225 the $\mathrm{mtp}^{-{ }^{-}}$and apoC2 $2^{-/}$mutant lines. $\mathrm{mtp}^{-/-}$mutants produce smaller IDL and LDL-like particles from

226 the earliest stages of development, and apoC2--- mutants show a VLDL peak that persists

227 throughout development (Fig. 3c,f). Consistent with the data from $\mathrm{mt}^{-\mathrm{f}^{-}}$mutants, pharmacological

228 treatment with a potent MTP inhibitor (Lomitapide) effectively blocks the production of new VLDL

229 particles (Fig. 3d,g) leading to the accumulation of lipolyzed species such as IDL and LDL.

230 Consistent with the mammalian literature, a robust post prandial response to a high lipid meal

231 (egg yolk emulsion) was observed in the distribution of ABCL subclasses of larval zebrafish (Fig.

$2323 e, \mathrm{~h})$. After fasting (48 h), there is significant depletion of VLDL and enrichment of LDL, consistent

233 with cessation of VLDL production due to limited nutrient availability. A subsequent high-fat meal

234 produces a significant increase in the ZM band, and progressive depletion of LDL.

Electrophoretic mobility correlates well with lipoprotein density and size

Electrophoretic mobility in Native-PAGE is a function of both size and charge, so it is

238 important to evaluate whether differences in migration truly reflect different lipoprotein sizes or if

239 they are the result of differentially charged lipoproteins. Density gradient ultracentrifugation (DGUC)

240 is the gold standard for discerning different subclasses of ABCLs, as larger lipoprotein classes are

241 more buoyant resulting from their large lipid core. To evaluate concordance between DGUC and the

242 LipoGlo assays, we developed a DGUC protocol (based on the method described by Yee et al., [40])

243 to separate pooled larval homogenate into density fractions. We then subjected fractions to (i)

244 LipoGlo electrophoresis to characterize their electrophoretic mobility, (ii) a plate read assay to

245 quantify ApoB-NanoLuc levels, and (iii) negative-staining electron microscopy to visualize particle

246 size directly [41] (Fig. 4). Importantly, denser fractions showed higher electrophoretic mobility and

247 smaller particle sizes across all genotypes, demonstrating that electrophoretic mobility is a reliable

248 method for differentiating $\mathrm{ABCL}$ classes and can be used as a proxy to estimate particle size and

249 density.

250

\section{LipoGlo-Microscopy reveals whole-organism ABCL localization}

252 The transparency of larval zebrafish offers the unique opportunity to perform whole-mount 

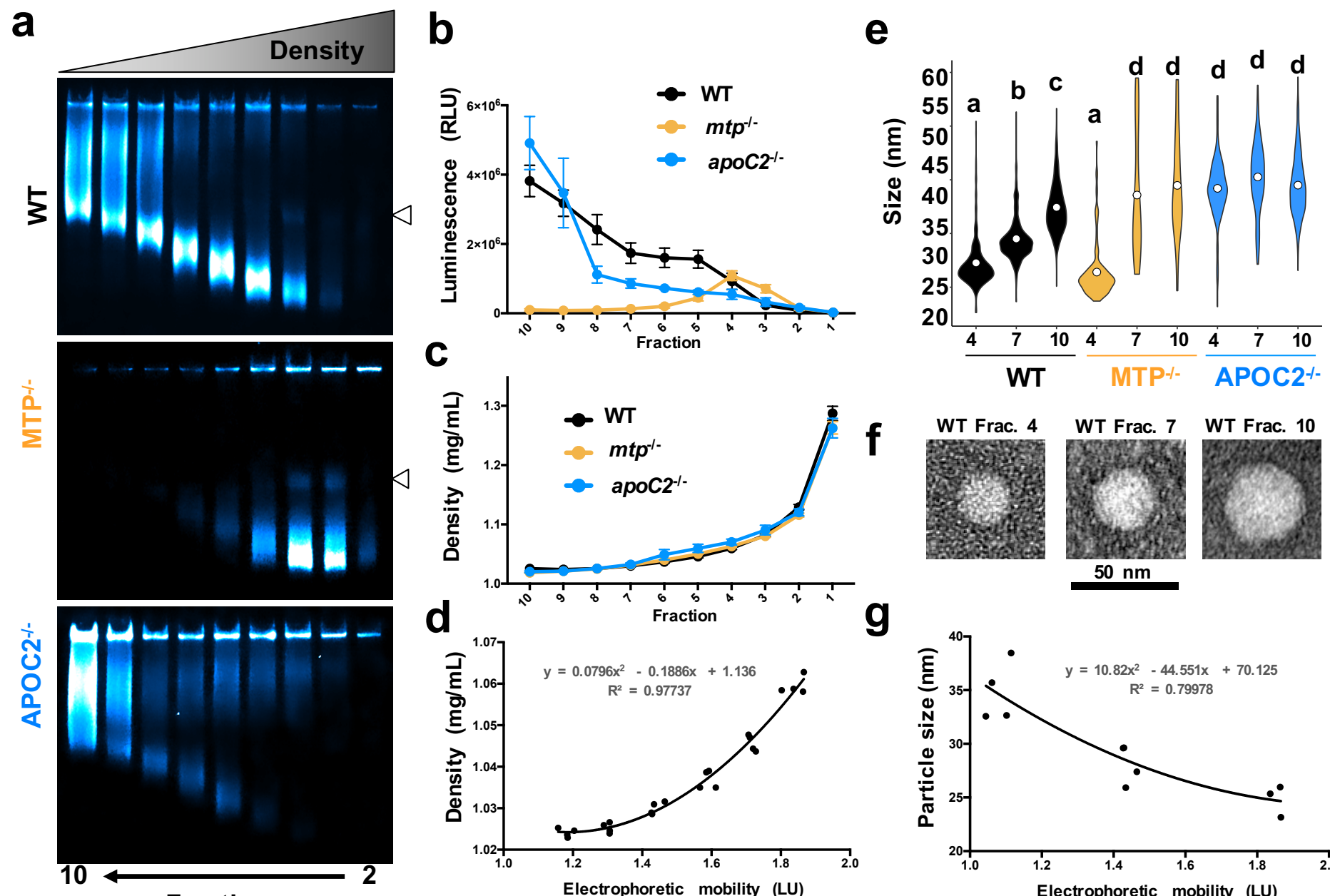

Fraction
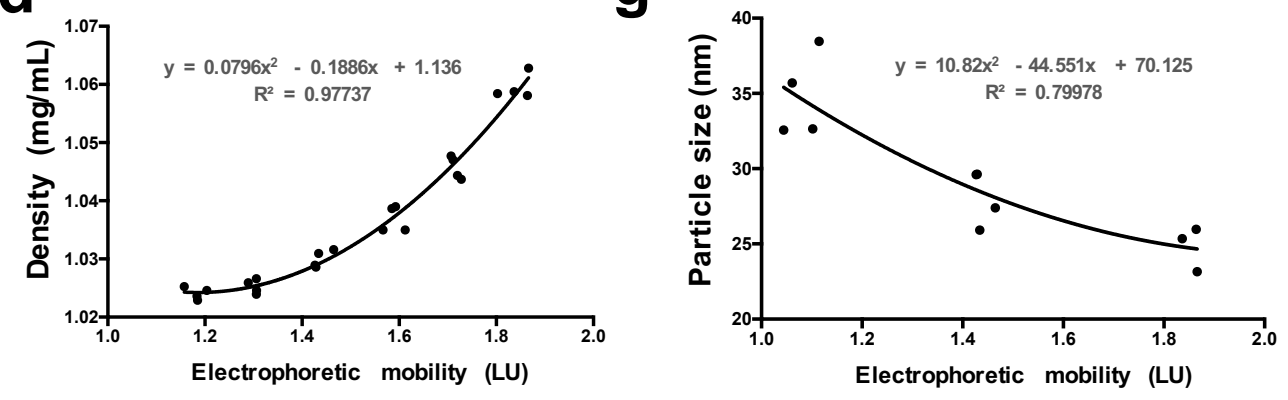

Figure 4:

Concordance between LipoGlo electrophoresis and classical ABCL size characterization techniques. DGUC was performed on pooled larval homogenate (4 dpf) from WT, $\mathrm{mtp}^{-/}$, and $\mathrm{apoC} \mathrm{C}^{-1}$, and separated into 10 equal fractions of approximately $500 \mu \mathrm{L}$ each by drip-elution (dense bottom fractions eluted first). (a) Fractions 2-10 were subjected to Native-PAGE, and denser fractions showed higher electrophoretic mobility. Some fractions show a faint lower mobility band (indicated at right by white arrowhead), possibly indicative of lipoprotein dimerization. (b) A plate-based assays of NanoLuc activity revealed the expected enrichment of VLDL in apoC2 $\%$ mutants, and enrichment of LDL in $m t^{-/}$mutants (confirming results reported in Fig. 3b). (c) A refractometer (Bausch and Lomb) was used to determine the refractive index of each fraction and density was calculated via the formula $\mathrm{D}=3.3508 \times \mathrm{RI}-3.4675$. DGUC showed highly reproducible density profiles between replicates and genotypes. (d) The density of WT fractions 4-9 was plotted as a function of peak electrophoretic mobility for that fraction, and the second order polynomial function $(y=0.0796 \times 2-0.1886 x+1.136)$ was able to represent this relationship with remarkable accuracy $\left(R^{2}\right.$ $=0.97737$ ) indicating that electrophoretic mobility is a useful proxy for lipoprotein density. (e) Fractions 4,7 , and 10 were subjected to negative-staining electron microscopy to directly visualize the size of particles in each fraction. In the wild type samples, the average particle diameter was $24.7 \pm 5.6,29.0 \pm 4.1$, and $34.9 \pm 4.7 \mathrm{~nm}$ for fractions 4,7 , and 10 respectively. There was no significant difference in particle size between fraction 4 of the wild-type and $m t^{-/}$mutant samples (average diameter of $23.2 \pm 6.6 \mathrm{~nm}$ ). Particles were nearly undetectable in fractions 7 and 10 in the $\mathrm{mtp}^{-/}$-mutant sample so particle diameter shows enormous variability. ABCLs in each apocC2- mutant fraction were significantly larger than all WT fractions, with diameters of $39.0 \pm 8.0,40.9 \pm 7.2$, and $39.1 \pm 5.9 \mathrm{~nm}$ respectively ( $n \approx 170$, Welch's ANOVA $p<0.0001$, Games-Howell $p<.0001$ ). (f) Representative images of lipoproteins from the three wild-type fractions are shown. (g) The second-order polynomial function $y=10.82 \times 2-44.551 x+70.125$ approximated the relationship between electrophoretic mobility and density in wild-type samples with reasonable accuracy $\left(R^{2}=0.79978\right)$. Results represent pooled data from four independent experiments. 
253 imaging, which has enabled us to perform the first characterization of changes in ABCL localization

254 throughout an intact organism. The same developmental, genetic, dietary, and pharmacological

255 manipulations described above (Figs. 2-3) were performed, but rather than being homogenized,

256 larvae were fixed in 4\% paraformaldehyde (PFA) for $3 \mathrm{~h}$ at room temperature, washed in PBS-

257 tween, and mounted in low-melt agarose [42] supplemented with Nano-Glo substrate solution.

258 Mounted larvae were imaged in a dark room on a Zeiss Axiozoom V16 equipped with a Zeiss

259 AxioCam MRm set to collect a single brightfield exposure followed by multiple exposures with no

260 illumination (chemiluminescent imaging).

261 The differences between WT, $m t p^{-/-}$, and apoC2 $2^{--}$mutants were most apparent at $6 \mathrm{dpf}$ (Fig.

262 5a). At this stage, the yolk is depleted and larvae are in a fasted state as no exogenous food has

263 been provided. In WT larvae, signal is quite low throughout the body, but is clearly visible in the

264 lipoprotein-producing tissues (liver and intestine). We observed a previously undescribed

265 association of ApoB with the spinal cord (Fig. 5b, and Supplementary Fig. 5a) as evidenced by

266 colocalization with the central-nervous system marker Tg(Xla.Tubb2:mApple-CAAX). This reporter

267 uses the tubulin beta-2 promoter from $X$. Laevis to drive a membrane-targeted mApple fluorophore

268 specifically in the CNS. A dorsal view revealed enrichment of NanoLuc signal in particular brain regions

269 (Fig. 5c), which we hypothesize may correspond to the brain ventricle. In $m t p^{-/-}$mutants, ApoB is

270 very low outside of the lipoprotein-producing tissues, consistent with defects in loading ApoB with

271 lipid to form a secretion-competent $\mathrm{ABCL}$. ApoC2 $2^{--}$mutants show remarkably high signal throughout

272 the body, consistent with their inability to process and turnover lipoproteins (Fig. 5a).

273 Images were quantified by creating separate regions of interest for the viscera, trunk, and

274 head regions (Supplementary figure 5c) and comparing the relative levels of NanoLuc signal in each

275 of these areas. During development, signal was initially highly enriched in the visceral region, which

276 contains the yolk and YSL, and then gradually increases in the trunk and head regions (Fig. 5d,g).

277 This is consistent with the vectorial transport of lipid from the YSL to the circulatory system and

278 peripheral tissues. The distribution of $A p o B$ between these three regions was not significantly

279 changed in apoC2-/- mutants, whereas $\mathrm{mtp}^{-/-}$mutants showed enrichment in the viscera and 
a

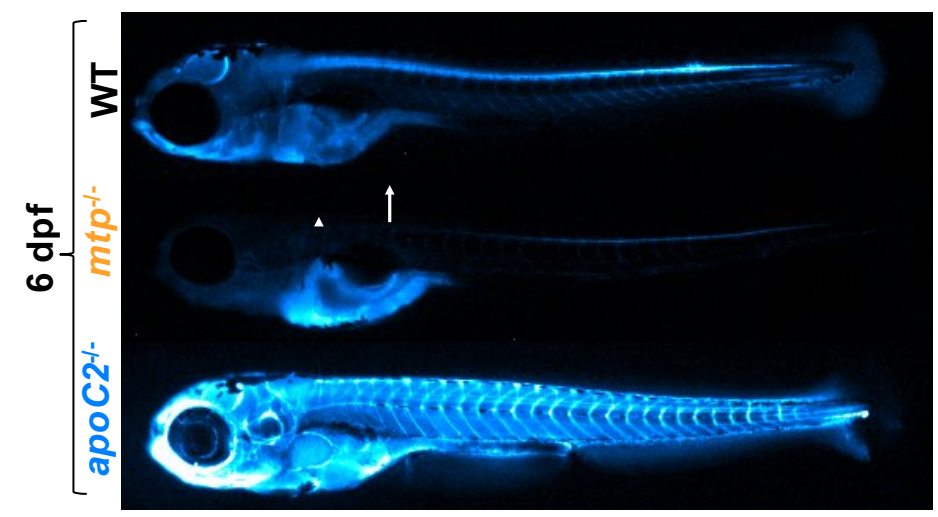

b

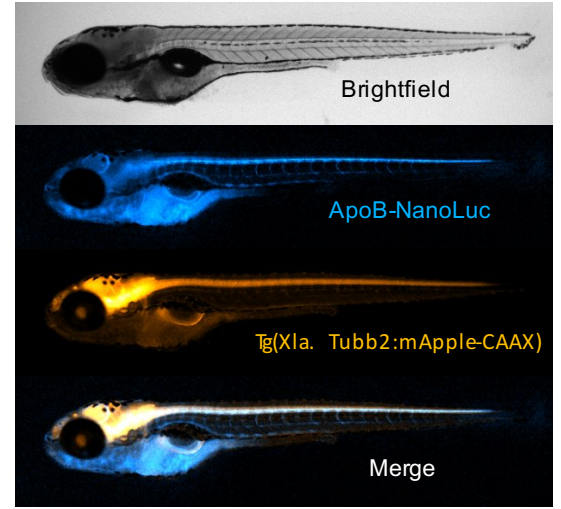

C

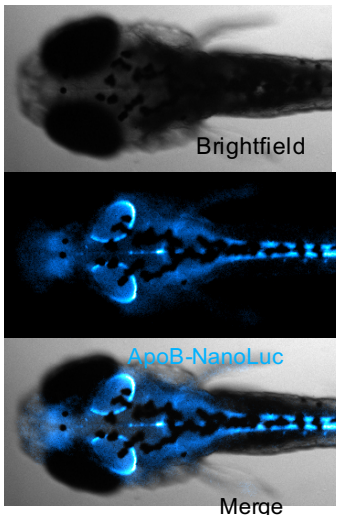

e
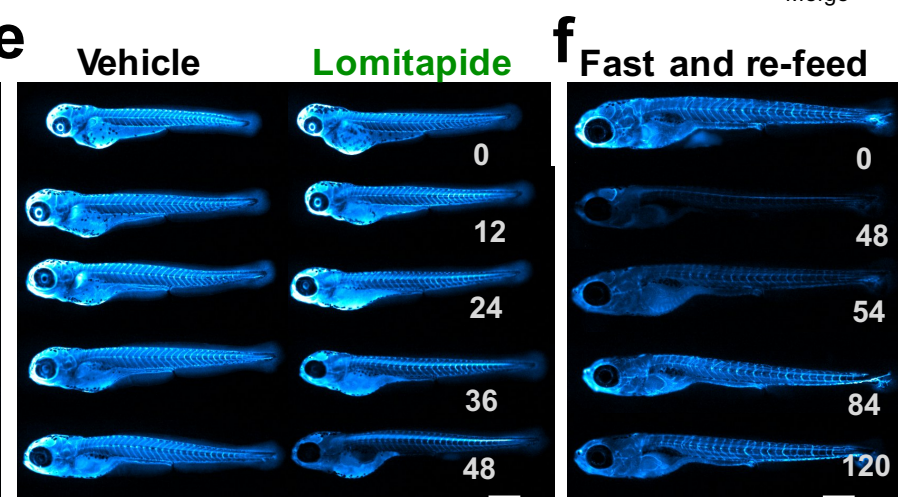

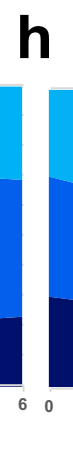

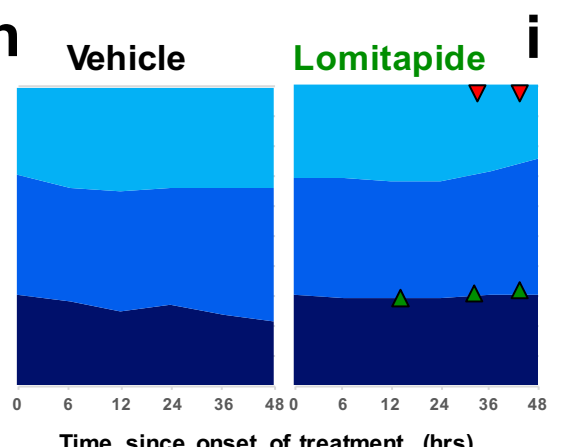

Time since onset of treatment (hrs)

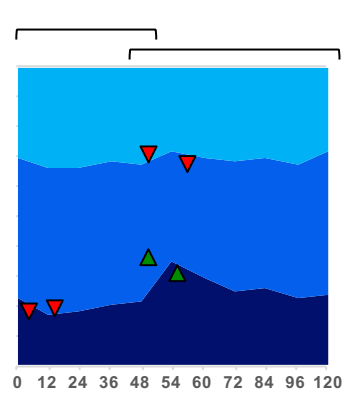

Time since onset of fast (hrs)

Figure 5:

Whole-mount imaging of $A B C L$ localization using LipoGlo chemiluminescent microscopy. (a) Representative images of $A B C L$ localization patterns from analysis of 15 larvae per genotype from WT, $m t p^{-/}$, and apoC2 ${ }^{-/}$genotypes (6 dpf). The white arrow and arrowhead mark the larval intestine and liver respectively. (b) LipoGlo signal colocalizes with the central nervous system marker Tg(Xla. Tubb2-mApple-CAAX), quantification in Supplementary Figure 5. (c) LipoGlo signal localized to subregions of the CNS. (d-f) Representative images and (g-i) quantification of ABCL localization across developmental, genetic, pharmacological, and dietary manipulations. Upward-facing arrowheads (green) indicate significant enrichment of that species at that time point compared to controls, and downward-facing arrowheads (red) indicate depletion. (g) Signal localization at each day of larval development in WT $(n=15$, Welch's ANOVA $p<0.0001$ for each region over time $), m t p^{-/}(n=15$, Two-way robust ANOVA $p<0.001$ for all regions, Games-Howell $p<0.001)$, and $a p o C 2^{--}(n=15$, Two-way robust ANOVA was not significant for any region) genetic backgrounds. (h) Signal localization from 3-5 dpf in larvae treated with $10 \mu \mathrm{M}$ lomitapide or vehicle control ( $\mathrm{n}=15$, Two-way robust ANOVA $p<0.001$ for head and viscera, Games-Howell $p<.0001$ ). (i) Subclass abundance from 10-15 dpf in larvae subjected to a fasting and re-feeding paradigm. The first bracket delineates changes relative to time 0 (the onset of the fasting period), and the second bracket delineates changes relative to time point 48 (the onset of the re-feeding period) ( $n=15$, Welch's ANOVA p<0.0001 for each region, Games-Howell $p<.005)$. Supplementary Figure 4 displays standard deviations for panels g-i. Results represent pooled data from three independent experiments, " $n$ " denotes number of samples per data point. Body regions were defined as outlined in Supplementary Figure 5. Scale bars $=500 \mu \mathrm{m}$. 
280 depletion in the peripheral tissues at all time points (Fig. 5d,g). Results were also grouped by region

281 to facilitate comparison of each class between genotypes (Supplementary Fig. 4).

\section{LipoGlo assays reveal Pla2g12b as an important regulator of ABCL homeostasis}

In an effort to identify novel regulators of the ABCL profile using the LipoGlo system, we

285 screened through several mutant lines from the zebrafish mutation project [43] that had predicted

286 mutations in genes involved in lipid metabolic pathways. Larvae homozygous for an essential splice

287 site mutation (sa659) in phospholipase A2 Group XII B (pla2g12b) showed perturbations in their

288 ABCL profile (Fig. 6). Homozygous mutant larvae exhibited lower levels of ApoB at multiple stages

289 (Fig. 6a), and also appeared to have defects in lipoprotein secretion as evidenced by enrichment of

290 visceral ApoB-NanoLuc levels (Fig. 6b,e). However, the most striking defect in pla2g12b-/ mutant

291 larvae is a pronounced change in the ABCL size distribution. Even at $1 \mathrm{dpf}$, significant accumulation

292 of small lipoproteins in the size range of LDL, and depletion of the larger particle classes, were

293 evident (Fig. 5c,d).

294

295 DISCUSSION:

\section{The LipoGlo reporter does not disrupt lipoprotein homeostasis}

When generating a fusion protein, it is essential to evaluate whether introduction of the tag

299 disrupts native protein function. This is particularly important in the case of tagged lipoproteins, as

300 these particles have a complex life cycle that involves interaction with numerous cell and tissue types.

301 To evaluate whether the NanoLuc tag disrupted lipoprotein homeostasis, LipoGlo larvae were

302 subjected to various genetic, dietary, and pharmacological manipulations known to affect the lipoprotein

303 profile. Detailed characterization of the lipoprotein profile in response to each of these stimuli revealed

304 the expected results in each case. These results validate that NanoLuc-tagged lipoproteins exhibit all of

305 the central hallmarks of endogenous ABCLs, including MTP-dependent maturation, APOC2-dependant

306 lipolysis, responsiveness to nutrient availability, and expected density and size distributions. 

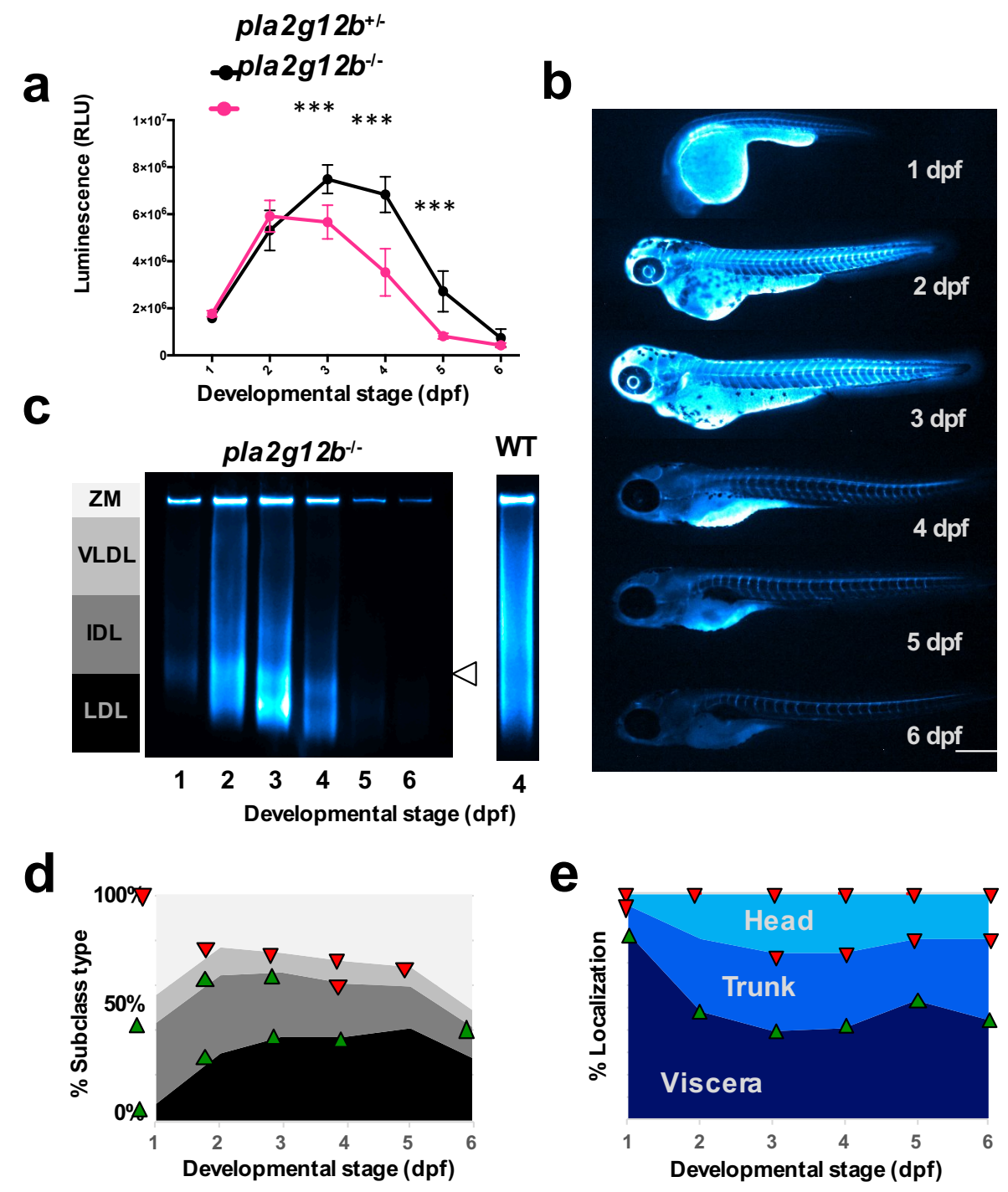

\section{Figure 6:}

LipoGlo reveals profound alterations in the ABCL profile in pla2g12 $\boldsymbol{b}^{-/-}$mutant larvae. (a) Comparison of LipoGlo signal between pla $2 \mathrm{~g} 12 \mathrm{~b}^{-/}$and pla $2 \mathrm{~g} 12 \mathrm{~b}^{+/}$siblings during larval development $(1-6 \mathrm{dpf})$ ( $\mathrm{n} \approx 11$, Two-way robust ANOVA p<0.0001 for genotype and stage, Games-Howell $p<.0001)$. (b) Representative images $(n=15)$ of ABCL localization collected by LipoGlo chemiluminescent imaging throughout development (1-6 dpf), and (e) quantification of percent localization into previously described subregions ( $n=15$, Two-way robust ANOVA $p<0.001$ for all regions, Games-Howell $p<0.0001$ ). (c) Representative gel $(\mathrm{n}=4)$ showing production of abnormally small lipoproteins (white arrowhead) with a lane of the 4 dpf WT profile (reproduced from Fig. 3a) provided for reference. (d) Quantification of LipoGlo emission pattern from LipoGlo electrophoresis in pla2g12b\% larvae (1-6 dpf). Upward-facing arrowheads (green) indicate significant enrichment of that species at that time point compared to WT, and downward-facing arrowheads (red) indicate depletion ( $n=9$, Two-way robust ANOVA p<0.001 for all species, Games-Howell $p<.01$ ). Results represent pooled data from three independent experiments, " $n$ " denotes number of samples per data point. Body regions were defined as outlined in Supplementary Figure 5. Scale bars $=500 \mu \mathrm{m}$. 
These observations establish a precedent for the use of ApoB-fusion proteins as a sensitive and

308 specific approach to monitor atherogenic lipoproteins in vivo. This approach can now be generalized to

309 function in essentially any model system expressing $A p o B$, and expanded to use alternative tags such

310 as fluorescent reporters for high-resolution imaging or affinity tags to study the lipoprotein interactome.

311 Potential applications of LipoGlo thus extend well beyond the study of lipoprotein abundance, size, and

312 localization using zebrafish.

\section{LipoGlo assays show excellent concordance with existing methods}

Atherogenic lipoproteins are the primary drivers of atherosclerotic cardiovascular disease, which has made lipoprotein profiling an essential technique in both clinical and research settings. Existing

317 methods to characterize ABCLs generally rely on large amounts of starting material (microliters of

318 plasma), use indirect measurements (e.g. lipid profiling), require expensive equipment and specialized

319 training (e.g. an ultracentrifuge or electron microscope), have a relatively low throughput capacity, and

320 are restricted to studying plasma lipoproteins. The LipoGlo reporter circumvents all of these issues and

321 engenders unprecedented speed, sensitivity, and tractability to the study of the lipoprotein profile.

322 Importantly, these assay shows excellent concordance with traditional methods, as evidenced by the

323 tight correlation between particle size estimates measured by LipoGlo electrophoresis and both density

324 gradient ultracentrifugation and negative-staining electron microscopy.

325 One limitation of the LipoGlo electrophoresis method reported here is that it is unable to

326 resolve ABCLs above a certain size threshold, which are clustered together as the zero-mobility

327 fraction. Further research will thus be required to conclusively determine which lipoprotein species

328 are present within this band. For example, this band is highly enriched in response to a high-fat

329 meal. This observation is consistent with enrichment of intracellular ApoB and the largest lipoprotein

330 classes (chylomicrons and remnants), both of which should increase in response to high-fat diet. It is

331 interesting to note, however, that when lipoproteins are processed through tandem density gradient

332 ultracentrifugation and LipoGlo-electrophoresis, there is significant signal in the ZM fraction even in

333 the higher density fractions. This indicates that the ZM band is not exclusively composed of very

334 large triglyceride-rich lipoproteins, but also includes additional denser species. We suspect this may 
335 reflect the physiological level of lipoprotein aggregation, which has been implicated as yet another

336 determinant of cardiovascular disease risk.

338 The larval zebrafish is a powerful new system to study lipoprotein biology

339 The LipoGlo system is significantly more sensitive than existing lipoprotein characterization

340 assays. To illustrate this, we have performed extensive lipoprotein characterization on individual

341 zebrafish larvae. A single larva contains approximately one-thousand times less plasma (nanoliters

342 rather than microliters) than is traditionally used for lipoprotein profiling, yet this is more than enough

343 material to run multiple LipoGlo assays. The larval zebrafish has been used extensively to study

344 metabolic diseases including cardiovascular disease [44], but the inability to characterize the

345 atherogenic lipoprotein profile in this model presented a significant limitation. LipoGlo enabled

346 comprehensive characterization of the atherogenic lipoprotein profile throughout larval development,

347 which will serve as a valuable resource for the zebrafish research community. LipoGlo was also used to

348 demonstrate numerous similarities in lipoprotein processing between larval zebrafish and humans.

349 The larval zebrafish is also an unparalleled vertebrate system for high-throughput screening.

350 The simple plate-based LipoGlo-counting method enables processing of tens of thousands samples a

351 day, and is thus readily conducive to high-throughput genetic and small-molecule screening. The

352 LipoGlo-electrophoresis and imaging protocols can also achieve respectable throughput capacity on

353 the order of hundreds of samples per day, and can thus be used as tractable secondary screening

354 assays or for stand-alone small-scale screens. The confluence of LipoGlo and the zebrafish model

355 system create the first opportunity to perform unbiased screens for novel modulators of ABCLs,

356 enabling powerful unbiased discovery approaches to be applied to the field of lipoprotein biology.

358 Sensitive lipoprotein profiling may provide new insights into abetalipoproteinemia

359 Human mutations in the mtp gene result in severe reduction or complete lack of ABCLs, a

360 disease called abetalipoproteinemia. The $m t p^{\text {stl }}$ allele studied contains a single missense mutation in a

361 highly conserved residue (L475P) [32]. Although this is thought to result in production of a non-

362 functional protein, a true null allele would be expected to result in a complete lack of ABCLs. The $m t p^{\text {stl }}$ 
363 homozygous mutants are unequivocally able to produce and secrete a ABCLs (although they are

364 smaller and less abundant) early in development, although at the later stages ABCLs are essentially

365 undetectable. These observations suggest that the $m t p^{\text {stl }}$ allele is either a strong hypomorph, or that

366 ABCLs can be produced without the activity of Mtp. The complete lack of ABCLs later in development

367 occurs once rates of particle turnover and uptake begin to greatly exceed rates of production. This

368 observation highlights the LipoGlo system as a useful tool to study allelic series of $m t p$. By studying

369 different alleles at the earliest stages of development when rates of lipoprotein turnover are low, it may

370 be possible to distinguish between true null alleles and varying degrees of hypomorphic mutations.

371 Such information would be useful in predicting the severity of different abetalipoprotienemia mutations

372 in humans.

373 Additionally, the $m t p^{-/}$mutants produce a distinct bimodal peak of small (LDL-like) ABCLs (Fig.

$3743 a-c)$. This pattern warrants further investigation, but may indicate that these alleles directly produce

375 small lipoproteins from the YSL, which are subsequently lipolyzed to produce the second peak. Further

376 study of this allele may provide insight into the specific functional domains of MTP that regulate the size

377 of nascent ABCLs.

379 Organ-level changes in lipoprotein localization

380 The localization of atherogenic lipoproteins throughout an intact organism has not previously

381 been reported. Several observations were in line with our expectations, in that lipoproteins were clearly

382 visible in lipoprotein-producing tissues such as the liver, intestine, and yolk-syncytial layer. Lipoproteins

383 were also clearly evident throughout the circulatory system as expected. However, one of the most

384 striking patterns of lipoprotein localization is the chevron pattern outlining the somites in the trunk

385 region. We suspect this pattern may correspond to the myosepta [45], which include tendinous

386 structures connecting the body segments. Lipoproteins have previously been shown to accumulate in

387 tendons in cases of severe hyperlipidemia [46], but these images suggest that an unexpectedly large

388 fraction of ABCLs localize to these structures in a normal physiological state. The physiological

389 consequences of this association are still unknown, but suggest that the pool of non-circulating ABCLs

390 cannot be ignored in studies of whole-body energy homeostasis. 
In addition to this somite pattern, surprisingly strong LipoGlo signal was also observed in the

392 central nervous system (both the brain and spinal cord), colocalizing with the CNS marker

393 Tg(Xla.Tubb2:mapple-CAAX). While this pattern was evident throughout development, it was most

394 pronounced in larvae that have low levels of atherogenic lipoproteins throughout the body (such as

395 wild-type larvae at $6 \mathrm{dpf}$, or larvae that have been fasted or treated with lomitapide for 48 hours, Fig.

$3965 d-f)$. Further studies will be required to determine the precise brain regions and structures that interact

397 with ABCLs, but the localization pattern observed is strikingly similar to that of fluorescein (a fluorescent

398 dye) after it is injected into the larval zebrafish ventricle [47]. Previous work in mammals has shown that

399 although the blood-brain barrier expresses the LDL-receptor, levels of ApoB within the cerebrospinal

400 fluid are extremely low [48]. These observations suggest that ABCLs may be preferentially retained in

401 the ventricle as a protected source of lipid for the brain in states of nutrient scarcity.

$402 \quad$ Although atherogenic lipoproteins are most often studied in the bloodstream as a means of

403 evaluating cardiovascular disease risk, these particles interact with essentially every tissue in the body

404 and are involved in numerous processes such as development [49], vision [50], angiogenesis [32],

405 heart function [51], hematopoiesis [52], infection and immunity [53, 54], cancer [55], and diabetes [56].

406 LipoGlo is an essential tool for broadening the scope of atherogenic lipoprotein biology beyond the

407 current focus on circulating particles. Although the vast majority of NanoLuc protein appears to be

408 associated with intact ABCLs, we cannot eliminate the possibility that some of the protein is cleaved

409 from the particle and may localize independently of ABCLs. Thus these preliminary observations

410 warrant further investigation and validation with orthogonal techniques.

412 Characterization of lipoprotein abnormalities in pla2g12 $\boldsymbol{b}^{-/}$mutants

413 Phospholipase A2 group XII B (Pla2g12b) is a catalytically inactive member of the

414 phospholipase gene family. Although this gene lacks catalytic activity and has no other known

415 function, its high level of evolutionary conservation suggests it may have evolved a new function.

416 Previous studies in mice have shown that disruption of pla2g12b results in decreased secretion of

417 hepatic triglyceride and ApoB [57], as well as reduced levels of HDL-cholesterol [58], indicating that

418 this gene may play a role in lipoprotein secretion. LipoGlo revealed that pla2g $12 b^{-/-}$mutant larvae 
419 exhibited significantly lower levels of ApoB at multiple stages, and show enrichment of visceral

420 ApoB-NanoLuc levels, both of which are consistent with previously reported defects in lipoprotein

421 secretion. However, evaluation of the lipoprotein size distribution in pla2g12 $b^{-/-}$mutants revealed bias

422 towards production of small LDL-like particles, which has not been reported previously. Further

423 investigation will be required to determine whether smaller ABCLs are produced directly by the

424 YSL/liver, or if the decreased ABCL secretion rate results in rapid lipolysis of the few lipoproteins that

425 are successfully secreted. As Pla2g12b modulates both lipoprotein size and number, variants in this

426 gene may modulate risk for cardiovascular disease. Ongoing work is exploring both the mechanism of

427 action of this poorly understood protein, as well as the greater physiological repercussions of this

428 mutation.

430 Overview of present and future application of LipoGlo

431 Overall, this study provides several key insights. Firstly, covalent tags to ApoB enable highly

432 sensitive and specific monitoring of ABCLs without disrupting lipoprotein homeostasis. Secondly, the

433 larval zebrafish represents a powerful new model to study ABCLs, as developmental stages provide a

434 highly reproducible pattern of nutrient availability that shows human-like responses to genetic, dietary,

435 and pharmacological stimuli. Additionally, larval zebrafish have unparalleled tractability for genetic and

436 small-molecule screening as well as whole-organism imaging, facilitating the application of these

437 powerful discovery techniques to the field of lipoprotein biology. ABCLs also show significant patterns

438 of enrichment outside of the circulatory system (in association with the somite junctions and central

439 nervous system), laying the foundation for the continued study of extravascular ABCLs. Lastly,

440 pla2g12b is a highly conserved regulator of lipoprotein biogenesis that plays a central role in the

441 regulation of the lipoprotein size/subclass distribution. The LipoGlo system thus represents an essential

442 tool to expand our understanding of atherogenic lipoproteins and accelerate the discovery of new

443 approaches to combat atherosclerotic cardiovascular disease. 
447 ACKNOWLEDGEMENTS:

We would like to thank Promega Corp. for providing the NanoLuc plasmid, as well as sample

450 reagents and technical advice that were essential to assay development, as well as serving as a co-

451 sponsor for a local lipid research conference where this work was presented. We would also like to

452 thank Michael Sepanski for collecting the electron micrographs, Dr. Marnie Halpern for providing the

453 unpublished $\operatorname{Tg}($ Xla.Tubb2:mapple-CAAX) pan-neuronal marker line and valuable advice on the

454 manuscript, Dr. Yury Miller for providing the apoC2 mutant line, and the Sanger Institute Zebrafish

455 Mutation project for providing the pla2g12b mutant line (sa659). Support was also provided by the

456 National Institutes of Health (R01DK093399 [S.A.F.] and R01DK116079 [S.A.F.]), National Heart, Lung,

457 and Blood Institute (F31HL139338 [J.H.T]) and National Institute of General Medical Sciences

458 (R01GM63904 [S.C.E] \& [S.A.F] and P30DK084567 [S.C.E.]). This content is solely the responsibility of

459 the authors and does not necessarily represent the official views of $\mathrm{NIH}$. Additional support for this work

460 was provided by the Carnegie Institution for Science endowment and the G. Harold and Leila Y.

461 Mathers Charitable Foundation (S.A.F).

\section{AUTHOR CONTRIBUTIONS:}

464 J.H.T. and S.A.F. conceived and designed the project, and met frequently to discuss results, plan

465 experiments, and troubleshoot protocols. S.C.E. provided critical reagents and expertise to design and

466 synthesize the TALENs used to create the LipoGlo fish line. J.H.T. executed the experiments, analyzed

467 the results, and wrote the original draft of the paper. J.H.T., S.C.E. and S.A F. revised, edited, and

468 approved the final submitted version of the manuscript.

471 DECLARATION OF INTERESTS:

472 The Authors declare no competing interests. 
475 REFERENCES:

476

477 1. Tabas, I., K.J. Williams, and J. Boren, Subendothelial lipoprotein retention as the initiating 478 process in atherosclerosis: update and therapeutic implications. Circulation, 2007. 116(16):

479 p. $1832-44$.

480 2. WHO. The top 10 causes of death. Fact Sheets [Web Page] 2018 05/24/2018 [cited 2018

481 09/25/2018]; Available from: http://www.who.int/news-room/fact-sheets/detail/the-

482 top-10-causes-of-death.

483 3. Stone, N.J., et al., 2013 ACC/AHA guideline on the treatment of blood cholesterol to reduce

484 atherosclerotic cardiovascular risk in adults: a report of the American College of

485 Cardiology/American Heart Association Task Force on Practice Guidelines. Circulation, 2014.

$486 \quad 129(25$ Suppl 2): p. S1-45.

487 4. McQueen, M.J., et al., Lipids, lipoproteins, and apolipoproteins as risk markers of myocardial

488 infarction in 52 countries (the INTERHEART study): a case-control study. Lancet, 2008.

$489 \quad 372(9634):$ p. 224-33.

$490 \quad 5 . \quad$ Sniderman, A.D., et al., Discordance analysis of apolipoprotein $B$ and non-high density

491 lipoprotein cholesterol as markers of cardiovascular risk in the INTERHEART study.

492 Atherosclerosis, 2012. 225(2): p. 444-9.

493 6. $\quad$ Lawler, P.R., et al., Atherogenic Lipoprotein Determinants of Cardiovascular Disease and

494 Residual Risk Among Individuals With Low Low-Density Lipoprotein Cholesterol. J Am Heart

$495 \quad$ Assoc, 2017.6(7).

496 7. Rizzo, M. and K. Berneis, Low-density lipoprotein size and cardiovascular risk assessment.

497 QJM, 2006. 99(1): p. 1-14.

498 8. Austin, M.A., et al., Low-density lipoprotein subclass patterns and risk of myocardial

499 infarction. JAMA, 1988. 260(13): p. 1917-21. 
5009 9. Shim, H., et al., A multivariate genome-wide association analysis of 10 LDL subfractions, and

501 their response to statin treatment, in 1868 Caucasians. PLoS One, 2015. 10(4): p. e0120758.

502 10. Chasman, D.I., et al., Forty-three loci associated with plasma lipoprotein size, concentration,

503 and cholesterol content in genome-wide analysis. PLoS Genet, 2009. 5(11): p. e1000730.

504 11. Berneis, K.K. and R.M. Krauss, Metabolic origins and clinical significance of LDL

505 heterogeneity. J Lipid Res, 2002. 43(9): p. 1363-79.

506 12. Rajman, I., et al., LDL particle size: an important drug target? Br J Clin Pharmacol, 1999.

$507 \quad$ 48(2): p. $125-33$.

508 13. Jacobson, T.A., Opening a new lipid "apo-thecary": incorporating apolipoproteins as potential

509 risk factors and treatment targets to reduce cardiovascular risk. Mayo Clin Proc, 2011.

$510 \quad$ 86(8): p. 762-80.

511 14. Xiao, C., et al., Pharmacological Targeting of the Atherogenic Dyslipidemia Complex: The Next

$512 \quad$ Frontier in CVD Prevention Beyond Lowering LDL Cholesterol. Diabetes, 2016. 65(7): p.

$513 \quad 1767-78$.

514 15. Liu, C., et al., Apoc2 loss-of-function zebrafish mutant as a genetic model of hyperlipidemia.

515 Dis Model Mech, 2015. 8(8): p. 989-98.

$516 \quad$ 16. Schlegel, A., Zebrafish Models for Dyslipidemia and Atherosclerosis Research. Front

$517 \quad$ Endocrinol (Lausanne), 2016. 7: p. 159.

$518 \quad$ 17. O'Hare, E.A., et al., Disruption of ldlr causes increased LDL-c and vascular lipid accumulation

519 in a zebrafish model of hypercholesterolemia. J Lipid Res, 2014. 55(11): p. 2242-53.

520 18. Mahley, R.W., Central Nervous System Lipoproteins: ApoE and Regulation of Cholesterol

521 Metabolism. Arterioscler Thromb Vasc Biol, 2016. 36(7): p. 1305-15.

522 19. Rouault, M., et al., Novel mammalian group XII secreted phospholipase A2 lacking enzymatic

523 activity. Biochemistry, 2003. 42(39): p. 11494-503.

524 20. Elovson, J., et al., Plasma very low density lipoproteins contain a single molecule of

525 apolipoprotein B. J Lipid Res, 1988. 29(11): p. 1461-73. 
526 21. Fisher, E., E. Lake, and R.S. McLeod, Apolipoprotein B100 quality control and the regulation

527 of hepatic very low density lipoprotein secretion. J Biomed Res, 2014. 28(3): p. 178-93.

528 22. Kane, J.P., D.A. Hardman, and H.E. Paulus, Heterogeneity of apolipoprotein B: isolation of a new species from human chylomicrons. Proc Natl Acad Sci U S A, 1980. 77(5): p. 2465-9.

530 23. Davidson, N.O. and G.S. Shelness, APOLIPOPROTEIN B: mRNA editing, lipoprotein assembly, 531 and presecretory degradation. Annu Rev Nutr, 2000. 20: p. 169-93.

532 24. Otis, J.P., et al., Zebrafish as a model for apolipoprotein biology: comprehensive expression analysis and a role for ApoA-IV in regulating food intake. Dis Model Mech, 2015. 8(3): p. 295-

$534 \quad 309$.

535 25. Hussain, M.M., et al., Amino acids 430-570 in apolipoprotein $B$ are critical for its binding to 536 microsomal triglyceride transfer protein. J Biol Chem, 1998. 273(40): p. 25612-5.

537 26. Boren, J., et al., The molecular mechanism for the genetic disorder familial defective 538 apolipoprotein B100. J Biol Chem, 2001. 276(12): p. 9214-8.

539 27. Hall, M.P., et al., Engineered luciferase reporter from a deep sea shrimp utilizing a novel $540 \quad$ imidazopyrazinone substrate. ACS Chem Biol, 2012. 7(11): p. 1848-57.

541 28. Shin, J., J. Chen, and L. Solnica-Krezel, Efficient homologous recombination-mediated genome 542 engineering in zebrafish using TALE nucleases. Development, 2014. 141(19): p. 3807-18.

543 29. Miyares, R.L., V.B. de Rezende, and S.A. Farber, Zebrafish yolk lipid processing: a tractable $544 \quad$ tool for the study of vertebrate lipid transport and metabolism. Dis Model Mech, 2014. 7(7):

$545 \quad$ p. $915-27$.

546 30. Hussain, M.M., J. Shi, and P. Dreizen, Microsomal triglyceride transfer protein and its role in 547 apoB-lipoprotein assembly. J Lipid Res, 2003. 44(1): p. 22-32.

548 31. Jong, M.C., M.H. Hofker, and L.M. Havekes, Role of ApoCs in lipoprotein metabolism: 549 functional differences between ApoC1, ApoC2, and ApoC3. Arterioscler Thromb Vasc Biol, $550 \quad$ 1999. 19(3): p. $472-84$. 
551 32. Avraham-Davidi, I., et al., ApoB-containing lipoproteins regulate angiogenesis by modulating

552 expression of VEGF receptor 1. Nat Med, 2012. 18(6): p. 967-73.

553 33. Cuchel, M., et al., Inhibition of microsomal triglyceride transfer protein in familial

554 hypercholesterolemia. N Engl J Med, 2007. 356(2): p. 148-56.

555 34. Carten, J.D., M.K. Bradford, and S.A. Farber, Visualizing digestive organ morphology and

556 function using differential fatty acid metabolism in live zebrafish. Dev Biol, 2011. 360(2): p.

$557 \quad 276-85$.

$558 \quad 35 . \quad$ Feingold, K.R. and C. Grunfeld, Introduction to Lipids and Lipoproteins, in Endotext, L.J. De

559 Groot, et al., Editors. 2000: South Dartmouth (MA).

560 36. Singh, Y., et al., A rapid 3\% polyacrylamide slab gel electrophoresis method for high through

561 put screening of LDL phenotype. Lipids Health Dis, 2008. 7: p. 47.

562 37. Hoefner, D.M., et al., Development of a rapid, quantitative method for LDL subfractionation

563 with use of the Quantimetrix Lipoprint LDL System. Clin Chem, 2001. 47(2): p. 266-74.

564 38. Sato, A., et al., Angiotensin II induces the aggregation of native and oxidized low-density

565 lipoprotein. Eur Biophys J, 2018. 47(1): p. 1-9.

566 39. Tiwari, S. and S.A. Siddiqi, Intracellular trafficking and secretion of VLDL. Arterioscler

567 Thromb Vasc Biol, 2012. 32(5): p. 1079-86.

568 40. Yee, M.S., et al., Lipoprotein separation in a novel iodixanol density gradient, for composition, 569 density, and phenotype analysis. J Lipid Res, 2008. 49(6): p. 1364-71.

570 41. Garewal, M., L. Zhang, and G. Ren, Optimized negative-staining protocol for examining lipid-

571 protein interactions by electron microscopy. Methods Mol Biol, 2013. 974: p. 111-8.

572 42. Westerfield, M., The zebrafish book: a guide for the laboratory use of zebrafish (Danio rerio).

573 2007: University of Oregon press.

574 43. Kettleborough, R.N., et al., A systematic genome-wide analysis of zebrafish protein-coding 575 gene function. Nature, 2013. 496(7446): p. 494-7. 
576 44. Chico, T.J., P.W. Ingham, and D.C. Crossman, Modeling cardiovascular disease in the 577 zebrafish. Trends Cardiovasc Med, 2008. 18(4): p. 150-5.

578 45. Charvet, B., et al., Development of the zebrafish myoseptum with emphasis on the 579 myotendinous junction. Cell Tissue Res, 2011. 346(3): p. 439-49.

$580 \quad$ 46. Tsouli, S.G., et al., Regression of Achilles tendon thickness after statin treatment in patients with familial hypercholesterolemia: an ultrasonographic study. Atherosclerosis, 2009.

583 47. Henson, H.E., et al., Functional and genetic analysis of choroid plexus development in 584 zebrafish. Front Neurosci, 2014. 8: p. 364.

585 48. Dehouck, B., et al., A new function for the LDL receptor: transcytosis of LDL across the blood586 brain barrier. J Cell Biol, 1997. 138(4): p. 877-89.

587 49. Neumann, S., et al., Mammalian Wnt3a is released on lipoprotein particles. Traffic, 2009. $588 \quad$ 10(3): p. 334-43.

589 50. Pikuleva, I.A. and C.A. Curcio, Cholesterol in the retina: the best is yet to come. Prog Retin Eye $590 \quad$ Res, 2014. 41: p. 64-89.

591 51. Yu, X., et al., Inhibition of cardiac lipoprotein utilization by transgenic overexpression of 592 Angptl4 in the heart. Proc Natl Acad Sci U S A, 2005. 102(5): p. 1767-72.

593 52. Liu, C., et al., Lipoprotein lipase regulates hematopoietic stem progenitor cell maintenance 594 through DHA supply. Nat Commun, 2018. 9(1): p. 1310.

595 53. Manifold-Wheeler, B.C., et al., Serum Lipoproteins Are Critical for Pulmonary Innate Defense 596 against Staphylococcus aureus Quorum Sensing. J Immunol, 2016. 196(1): p. 328-35.

597 54. Bashmakov, Y.K., et al., ApoB-containing lipoproteins promote infectivity of chlamydial 598 species in human hepatoma cell line. World J Hepatol, 2010. 2(2): p. 74-80.

$599 \quad 55 . \quad$ Borgquist, S., et al., Apolipoproteins, lipids and risk of cancer. Int J Cancer, 2016. 138(11): p. $600 \quad 2648-56$. 
601 56. Ley, S.H., et al., Association of apolipoprotein B with incident type 2 diabetes in an aboriginal 602 Canadian population. Clin Chem, 2010. 56(4): p. 666-70.

603 57. Guan, M., et al., Hepatocyte nuclear factor-4 alpha regulates liver triglyceride metabolism in 604 part through secreted phospholipase A(2) GXIIB. Hepatology, 2011. 53(2): p. 458-66.

605 58. Aljakna, A., et al., Pla2g12b and Hpn are genes identified by mouse ENU mutagenesis that 606 affect HDL cholesterol. PLoS One, 2012. 7(8): p. e43139.

607 59. Neff, K.L., et al., Mojo Hand, a TALEN design tool for genome editing applications. BMC 608 Bioinformatics, 2013. 14: p. 1.

609 60. Ma, A.C., et al., FusX: A Rapid One-Step Transcription Activator-Like Effector Assembly System 610 for Genome Science. Hum Gene Ther, 2016. 27(6): p. 451-63.

611 61. Petersen, L.K. and R.S. Stowers, A Gateway MultiSite recombination cloning toolkit. PLoS One, 2011. 6(9): p. e24531.

613 62. Rumsey, S.C., et al., Cryopreservation with sucrose maintains normal physical and biological properties of human plasma low density lipoproteins. J Lipid Res, 1992. 33(10): p. 1551-61.

615 63. Meeker, N.D., et al., Method for isolation of PCR-ready genomic DNA from zebrafish tissues. Biotechniques, 2007. 43(5): p. 610, 612, 614.

620 MATERIALS AND METHODS:

\section{Zebrafish husbandry and maintenance}

Adult zebrafish were maintained on a $14 \mathrm{~h}$ light - $10 \mathrm{~h}$ dark cycle and fed once daily with $\sim 3.5 \%$

624 body weight of Gemma Micro 500 (Skretting USA). All genotypes were bred into the wild-type AB

625 background. All assays were performed on larvae heterozygous for the ApoB-Nanoluc reporter unless

626 otherwise noted. To monitor the wild-type lipoprotein profile throughout larval development, pairwise

627 crosses were set up between wild-type AB adults and adults homozygous for the ApoB-NanoLuc 
628 reporter $\left(a p o B b .1^{\text {NLuc/NLuc }}\right)$. To characterize the lipoprotein profile of $m t p$ mutant larvae [32], pairwise

629 crosses were set up between $m t p^{\mathrm{st} / /+}$ and $m t p^{\mathrm{st} /++} ; a p o B b .1^{\mathrm{NLuc} / \mathrm{NLuc}}$ adults. To characterize the lipoprotein

630 profile of apoC2 mutant larvae [15], pairwise crosses were set up between apoC2 $2^{\text {sd38/sd38 and }}$

$631 a p o C 2^{\text {sd38/+ }} ; a p o B b .1^{\text {NLuc/+ }}$ adults and larvae positive for the NanoLuc reporter were selected for

632 analysis. To characterize the lipoprotein profile of pla2g12b mutant larvae [43], pairwise crosses were

633 set up between pla2g12 $b^{\text {sa659/sa659 }}$ and pla2g12 $b^{\text {sa659/+ }} ;$ apoBb. $1^{\text {NLuc/+ }}$ adults. To evaluate association

634 between the ABCLs and the central nervous system, adults homozygous for the ApoB-NanoLuc

635 reporter $\left(a p o B b .1^{\text {NLuc/NLuc }}\right)$ were crossed to adults heterozygous for the central nervous system marker

$636 \mathrm{Tg}$ (Xla.Tubb2:mapple-CAAX), and embryos were screened for mApple prior to fixation and mounting

637 (unpublished reagent provided by the Halpern Lab, c583). As zebrafish sex cannot be determined

638 during the larval stages, gender can be excluded as a variable. All procedures were approved by the

639 Carnegie Institution Animal Care and Use Committee (Protocol \#139).

640

641 Genome editing

642 Genome integration was achieved by co-injection of 500 pg of TALEN mRNA and 30 pg of

643 donor plasmid into 1-cell stage embryos (Supplementary Fig. 2a). Two pairs of TALENs were designed

644 and cloned that target a Bsrl restriction site just upstream of the endogenous stop codon of ApoBb.1

645 using the Mojo Hand design tool [59] and FusX assembly system [60]. TALENs were in-vitro

646 transcribed using the T3 mMessage mMachine kti (ThermoFisher Scientific, AM1348) and injected into

647 1-cell stage zebrafish embryos. Cutting efficiency was quantified by monitoring the loss of Bsrl

648 digestion as a result of TALEN nuclease activity, and found to be significantly higher in TALEN pair 2,

649 so this pair was used for genome integration efforts (Supplementary Fig. 2b). A donor plasmid was

650 cloned using 3-fragment MultiSite gateway assembly (Invitrogen, 12537-023) with a 5' entry element of

$651 \sim 500 \mathrm{bp}$ of the genomic sequence upstream of the ApoBb.1 stop codon, a middle-entry element

652 consisting of in-frame NanoLuc coding sequence, and a 3' element of $\sim 700$ bp of genomic sequence

653 downstream of the ApoBb.1 stop codon [61]. Injected embryos were raised to adulthood and progeny

654 were screened for NanoLuc activity and in-frame fusion of the NanoLuc reporter at the target locus

655 (Supplementary Fig. 2c). 
657 Preparation and storage of larval homogenate

Individual larvae are homogenized in a standard volume of ABCL stabilization buffer (100 $\mu \mathrm{L})$.

659 The ABCL stabilization buffer (see recipes) contains cOmplete Mini, EDTA-free Protease Inhibitor

660 Cocktail (Millipore-Sigma, 11836170001), pH buffer and calcium chelator (EGTA, pH 8), and

661 cryoprotectant [62] (Sucrose) to preserve sample integrity during homogenization (Supplementary Fig.

662 6). The buffer is made as a $2 x$ stock, and larvae are anesthetized in tricaine and placed into tubes in a

$66350 \mu \mathrm{L}$ volume and an equal volume of chilled $2 x$ buffer is then added just prior to homogenization. Low-

664 throughput homogenization can be achieved in $1.5 \mathrm{~mL}$ centrifuge tubes with disposable pellet-pestles

665 (Fisher scientific, 12-141-363). For high-throughput sample processing, larvae and ABCL stabilization

666 buffer are dispensed into individual wells of a 96-well non-skirted PCR-plate (USAScientific, \#1402-

667 9589), sealed with microSeal 'B' plate sealing film (Bio-Rad, msb1001), and homogenized in a

668 microplate-horn sonicator (Qsonica, Q700 sonicator with 431MPX microplate horn assembly). For

669 sonication, the plate was placed in the microplate horn filled with $17 \mathrm{~mm}$ of chilled RO water and

670 processed at $100 \%$ power for a total of 30 seconds, delivered as 2-second pulses interspersed with 1-

671 second pauses. Homogenate was stored on ice for immediate use, or frozen at $-20^{\circ} \mathrm{C}$ and thawed on

672 ice for later use.

\section{Quantification of ApoB-NanoLuc levels using a plate reader}

675 To quantify ApoB-NanoLuc levels, homogenate $(40 \mu \mathrm{L})$ was mixed with an equal volume of

676 diluted NanoLuc buffer (for specific dilution see recipes and technical note on NanoLuc buffer) in a 96-

677 well opaque white OptiPlate (Perkin-Elmer, 6005290). Black plates can be used as an alternative that

678 will significantly lower absolute signal intensity, but also reduce light contamination into adjacent wells.

679 The plate was read within 2 minutes of buffer addition using a SpectraMax M5 plate reader

680 (Moleculardevices) set to top-read chemiluminescent detection with a 500 ms integration time. This

681 plate-based assay has a wide linear range and long half-life (Supplementary Fig. 7a-c). However,

682 degree of pigmentation has a significant effect on signal intensity, so this variable should be accounted 
683 for with a standard curve or pigment-matched controls should be used as a baseline for comparison

684 (Supplementary Fig. 7d).

685

Quantification of lipoprotein size distribution with LipoGlo-electrophoresis

To quantify the electrophoretic mobility of ABCLs, $3 \%$ native polyacrylamide gels were cast in Bio-rad mini-protean casting rigs using $1 \mathrm{~mm}$ spacer plates and 10 -well combs (see recipes). Gels were

690 allowed to polymerize overnight at $4^{\circ} \mathrm{C}$ and used within $24 \mathrm{~h}$ of casting. Each gel included a migration

691 standard comprised of Di-I labeled human LDL (L3482, ThermoFisher Scientific) that was diluted in

692 cryoprotectant and stored in frozen aliquots (see recipes). Gels were assembled into mini-protean

693 electrophoresis rigs at $4^{\circ} \mathrm{C}$, filled with pre-chilled $1 \times \mathrm{x}$ TBE and pre-run at $50 \mathrm{~V}$ for 30 minutes to

694 equilibrate the gel prior to sample addition. $12 \mu \mathrm{L}$ of homogenate was then combined with $3 \mu \mathrm{L}$ of $5 \mathrm{x}$

695 load dye (see recipes), and $12.5 \mu \mathrm{L}$ of the resulting solution was loaded per well (which corresponds to

$69610 \%$ of the larval homogenate per lane). Gels were then run at $50 \mathrm{~V}$ for 30 minutes, followed by 125

697 volts for $2 \mathrm{~h}$.

698 Gels were imaged within $1 \mathrm{~h}$ of completion of the run. To image each gel, the thin glass short

699 plate was carefully separated from the front of the gel with a gel releaser wedge (see technical note on

700 hydrophobic coating of short plates). With the gel resting on the thick spacer plate, $1 \mathrm{~mL}$ of TBE

701 supplemented with $2 \mu \mathrm{L}$ of Nano-Glo substrate was gently pipetted onto the gel surface. The gel

702 imaging solution was spread evenly across the gel surface with a thin plastic film cut to the size of the

703 spacer plate (Staples, Sliding bar report covers). After a 5-minute equilibration, the gel was placed into

704 an Odyssey Fc (LI-COR Biosciences) gel imaging system (See technical note on gel imaging) and

705 imaged in the chemiluminescence channel for 2 minutes (NanoLuc detection) and then the 600 channel

706 for 30 seconds (Di-I LDL standard detection). Raw images were exported as zip files for further

707 analysis.

708 The provided gel quantification template (Supplemental File 1) can be used to bin the complex

709 lipoprotein size distribution into biologically relevant groups for analysis, and detailed instructions are

710 provided within the supplemental file. In short, each lane was converted to a plot profile in ImageJ, and 
711 divided into LDL, IDL, VLDL, and ZM bins based on migration relative to the Di-I standard, and pixel

712 intensity was summed within each bin for analysis.

713

714

\section{Larvae fixation and imaging}

716 To determine the whole-organism localization of ABCLs, intact larvae are anesthetized and

717 fixed in 4\% PFA (diluted in PBS) for $3 \mathrm{~h}$ at room temperature. Following fixation, larvae are rinsed 3

718 times for 15 minutes each in PBS-tween (PBS containing 0.1\% tween-20 detergent) and imaged within

$71912 \mathrm{~h}$ of fixation. Agarose for mounting is prepared by melting 0.1 grams of low-melting point agarose

720 (BP160-100, Fisher Scientific) in $10 \mathrm{mLs}$ of $1 \times$ TBE. Aliquots are maintained in the liquid state at $42^{\circ} \mathrm{C}$

721 in a heat block. Just prior to mounting, agarose aliquots were supplemented with 1\% Nano-Glo

722 substrate (furimazine). Fixed larvae are arrayed in droplets on a petri dish lid, and the excess liquid is

723 removed and quickly replaced with a $50 \mu \mathrm{L}$ droplet of low-melt agarose containing Nano-Glo substrate

724 (1\%). The sample is then oriented properly with a flexible poker until the agarose solidifies sufficiently to

725 hold the sample in place. This process was repeated for up to 15 larvae in parallel prior to imaging.

726 To image the ABCL localization, a Zeiss Axiozoom V16 microscope V16 equipped with a Zeiss

727 AxioCam MRm was set to 30x magnification, 2x2 binning and 2x gain (to increase sensitivity), and

728 programmed to collect a single brightfield exposure $(2.4 \mathrm{~ms}, 10 \%$ light intensity) followed by two

729 chemiluminescent imaging exposures (10 and 30 seconds, respectively) with no illumination to collect

730 the NanoLuc signal (See technical note on NanoLuc imaging). Images were quantified in ImageJ by

731 using the brightfield exposure to draw regions of interest (viscera, trunk, and head) and calculating the

732 NanoLuc intensity within each of those ROls for 30 second chemiluminescent exposure, unless

733 saturated pixels were detected in which case the 10 second exposure was used.

735 Density-gradient ultracentrifugation

736 A density gradient ultracentrifugation (DGUC) protocol was developed by adapting previously

737 published protocols using a 3-layer iodixanol gradient to function with smaller volumes of input sample

738 [40]. Individual larvae were sonicated in $100 \mu \mathrm{L}$ of sucrose-free ABCL buffer (see recipes) to avoid 
739 disruption of the density gradient with sucrose. 15 larvae were pooled per experiment into a single 1.5

$740 \mathrm{~mL}$ centrifuge tube and centrifuged for 5 minutes at 6,000 rcf to remove large cellular debris. $1 \mathrm{~mL}$ of

741 the resulting supernatant was transferred to a separate tube containing $500 \mu \mathrm{L}$ of Optiprep Density

742 gradient medium (D1556, Sigma-Aldrich) to yield a $20 \%$ iodixanol solution. A $9 \%$ iodizanol solution was

743 prepared by adding $1.5 \mathrm{~mL}$ of Optiprep to a $15 \mathrm{~mL}$ conical tube containing $8.5 \mathrm{~mL}$ HEPES-buffered

744 saline (HBS, see recipes), and a 12\% solution was prepared by mixing $2 \mathrm{~mL}$ Optiprep with $8 \mathrm{~mL}$ HBS.

745 A $4.9 \mathrm{~mL}$ Optiseal tube (formerly polyallomer, 362185, Beckman-Coulter) was then loaded with $1.5 \mathrm{~mL}$

746 of $9 \%$ iodixanol/HBS solution. This solution was carefully underlayered with $1.5 \mathrm{~mL}$ of the $12 \%$

747 iodixanol solution using a p1000 pipette fit with both the appropriate p1000 tip as well as a tapered gel

748 loading tip which functioned as a disposable plastic cannula (USA Scientific, 1252-0610). Finally, these

749 solutions were underlayered with $1.5 \mathrm{~mL}$ of the $20 \%$ iodixanol solution containing the zebrafish

750 homogenate. The tube was then topped up with HBS ( 500 uL) so that no air remained and sealed with

751 a cap. Balanced tubes were then loaded into a VTi65.2 rotor and centrifuged at 60,000 rpm in a

752 prechilled Beckman Optima XL $80 \mathrm{~K}$ Ultracentrifuge set to $4^{\circ} \mathrm{C}$ with maximum acceleration and

753 deceleration rates.

$754 \quad$ Following ultracentrifugation, density fractions were collected by carefully piercing the bottom of

755 the tube with a thumbtack, and drip-eluting the samples into 10 separate fractions of approximately 500

$756 \mu \mathrm{L}$ each. The refractive index of each fraction was determined using a Bausch and Lomb refractometer,

757 and used to calculate solution density using the formula density $=3.3508 \times$ (refractive index) -3.4675 .

758 Fractions were stored on ice or at $10^{\circ} \mathrm{C}$, and used within $24 \mathrm{~h}$ for a plate-based NanoLuc assay,

759 LipoGlo-electrophoresis, and negative-staining electron microscopy. Note that the high protein and

760 iodixanol content of fraction 1 (highest density) introduces artifacts in the native gel and was therefore

761 excluded, which allowed lane 1 to be dedicated to the Di-I LDL standard.

762

\section{Negative-staining electron microscopy}

764 Fractions 4, 7, and 10 from the DGUC experiments outlined above were subjected to negative-

765 staining electron microscopy [41]. 300-mesh copper grids coated with $10 \mathrm{~nm}$ formvar and $1 \mathrm{~nm}$ carbon

766 (Electron Microscopy Sciences, FCF300-Cu) were ionized using the glow discharge filament in a 
767 Denton Vacuum dv-502 evaporator at 75 mTorr for 30 seconds. Anti-capillary forceps were then used

768 to hold the grids in a humidified chamber, and $3 \mu \mathrm{L}$ of the sample was carefully placed on the surface of

769 the grid and incubated at room temperature for 10 minutes to allow the lipoproteins to adhere to the

770 grid. The grid was then rinsed in 5 droplets of RO-water and then finally 2 droplets of $2 \%$ uranyl

771 acetate, and touched lightly to a piece of filter paper to remove excess stain. Grids were imaged at

$77226,000 x$ magnification on a Tecnai 12 transmission electron microscope.

773

774 DNA extraction and Genotyping

775 Sonication of zebrafish larvae is a convenient method for highly-parallelized homogenization, as

776 a full plate (96 samples) can be processed simultaneously. However, this process shears DNA into

777 significantly smaller fragments, meaning longer amplicons will amplify less efficiently or not at all. To

778 circumvent this issue, genotyping protocols for this study were designed to use small amplicons (less

779 than $350 \mathrm{bp}$ ). If intact DNA is needed for downstream applications, the pellet-pestle method can be

780 used interchangeably with sonication.

781 DNA extraction of larval homogenate can be achieved with a modified version of the HotShot

782 DNA extraction protocol [63]. $10 \mu \mathrm{L}$ homogenate is transferred to a pcr tube/plate containing $10 \mu \mathrm{L}$ of

$783100 \mathrm{mM} \mathrm{NaOH}$, and heated at $95^{\circ} \mathrm{C}$ for 20 minutes. The solution was then neutralized with an equal

784 volume $(20 \mu \mathrm{L})$ of $100 \mathrm{mM}$ Tris $\mathrm{pH}$ 8, and either stored frozen $\left(-20^{\circ} \mathrm{C}\right)$ or used immediately as a

785 template for genotyping PCR (2 $\mu \mathrm{L}$ per reaction).

787 ApoBb.1-NanoLuc locus was genotyped using 3 primers with final concentrations as follows: $1 \mu \mathrm{M}$

788 primer $9, .2 \mu \mathrm{M}$ primer 10 , and $.8 \mu \mathrm{M}$ primer 11 . This ratio provides similar band intensity for the 113

789 bp product indicating presence of the WT allele, and the 161 bp product indicating NanoLuc fusion

790 allele $\left(\mathrm{T}_{\mathrm{a}}=57^{\circ} \mathrm{C}\right.$, extension time $\left.20^{\prime \prime}\right)$ in heterozygotes (only one band will amplify in homozygotes).

791 The mtp genotyping locus was amplified using primers 12 and $13\left(.5 \mu \mathrm{M}\right.$ each, $\mathrm{T}_{\mathrm{a}}=60^{\circ} \mathrm{C}$, extension

792 time 30"), and digested with 3 units of Avall restriction enzyme, which cuts the mutant (stII) allele. Wild-

793 type zebrafish should have a single $157 \mathrm{bp}$ band, homozygous mutants should have a shorter $129 \mathrm{bp}$

794 band, and heterozygotes should have both bands present (note the $28 \mathrm{bp}$ fragment is not usually 
795 detectable). The apoC2 genotyping locus was amplified using primers 14 and $15\left(.5 \mu \mathrm{M}\right.$ each, $\mathrm{T}_{\mathrm{a}}=$

$79657^{\circ} \mathrm{C}$, extension time $\left.30^{\prime \prime}\right)$, and digested with 3 units of Btsal restriction enzyme, which cuts the WT

797 allele but not the sd38 mutant allele. Wild-type zebrafish should have 102 and 45 bp bands,

798 homozygous mutants should have a single $147 \mathrm{bp}$ band, and heterozygotes should have all 3 bands

799 present. The pla2g12b genotyping locus was amplified using primers 16 and $17\left(.5 \mu \mathrm{M}\right.$ each, $\mathrm{T}_{\mathrm{a}}=57^{\circ} \mathrm{C}$,

800 extension time 30"), and digested with 3 units of Btsal restriction enzyme, which cuts the mutant

801 (sa659) allele. Wild-type zebrafish should have a single 150 bp band, homozygous mutants should

802 have a shorter $111 \mathrm{bp}$ band, and heterozygotes should have both bands present (note the $39 \mathrm{bp}$

803 fragment is not usually detectable).

804

\section{Technical notes and troubleshooting}

806

$\underline{\text { NanoLuc Buffer }}$

The NanoLuc enzyme is active in various buffers, but the key consideration is to ensure that

809 substrate is in excess. Manufacturer's instructions dictate that $1 \mathrm{~mL}$ of Buffer plus $20 \mu \mathrm{L}$ of substrate

810 solution constitutes a $2 x$ buffer, but we have found that this $2 x$ buffer can be diluted 4-fold in PBS and

811 the substrate remains in significant excess.

813 Hydrophobic coating of LipoGlo-electrophoresis plates

814 The most likely source of artifacts in the LipoGlo-electrophoresis protocol are from stretching or

815 distortion of the fragile 3\% polyacrylamide gel while removing the short plate from the gel. To

816 circumvent this issue, the short plates were coated on both sides with Rain-X original glass water

817 repellent (Rain-X, $3.5 \mathrm{oz}$. bottle). This hydrophobic coating greatly facilitates removal of the short plate

818 while leaving the undistorted gel resting on the spacer plate. This coating is semi-permanent, so it is

819 recommended that a set of coated short plates be dedicated for this purpose and reapplied with coating

820 as needed.

821 This hydrophobic coating also reduces friction between the short plate and the spacer plate, so

822 it is important that the plates are aligned properly in the casting frames and placed very gently in the 
823 casting stands. Too much pressure from the casting stand can cause the plates to slide out of

824 alignment and lead to leaking during casting.

825

826 Imaging of LipoGlo-electrophoresis gels

827 The Odyssey Fc offers sensitive signal detection as well as multi-color detection, and is

828 therefore ideal for imaging lipoprotein gels. However, if this equipment is not available, alternative gel

829 imaging systems or a sensitive camera are capable of imaging the gel as well, as the chemiluminescent

830 signal should be detectable by essentially any detector although the exposure time may need to be

831 increased to the order of minutes depending on the sensitivity of the detector. If simultaneous imaging

832 in chemiluminescent and fluorescent channels is not available, a large aliquot of zebrafish homogenate

833 (such as $6 \mathrm{dpf}$ larvae) can be pooled, aliquoted, frozen, and used as an alternative migration

834 normalization standard.

835

$836 \quad$ NanoLuc imaging

837 Essentially all background signal in this imaging paradigm comes from two sources: electrical

838 noise from the camera, and light contamination from the environment. Camera noise can be attenuated

839 by using an actively cooled camera and by enabling a blank-subtraction setting to eliminate hot pixels.

840 To reduce contaminating light from the environment, we recommend collecting images in a dark room

841 and shrouding the stage and/or microscope to prevent light from reaching the imaging path.

842 Additionally, we have found that the Zeiss Axiozoom V16 contains infrared emitters and detectors

843 within the imaging path, which result in very high background when long exposures are used. To

844 overcome this issue, we placed a Zeiss BG40 IR blocking filter in front of the camera which effectively

845 filtered the contaminating infrared light.

846

\section{Solutions/Recipes}

848

849 ABCL Stabilization Buffer (2x): For routine preparation of zebrafish homogenate

8501 CoMplete mini protease inhibitor tablet 
$400 \mu \mathrm{L} .5 \mathrm{M}$ EGTA $(\mathrm{pH} 8)$

852

1g Sucrose

853

Adjust volume to $5 \mathrm{~mL}$ with reverse osmosis (RO) water

854

855 Sucrose-free ABCL Stabilization Buffer (2x): For preparation of zebrafish homogenate for

856 ultracentrifugation

857 CoMplete mini protease inhibitor tablet

858

$400 \mu \mathrm{L} .5 \mathrm{M}$ EGTA $(\mathrm{pH} 8)$

859

Adjust volume to $5 \mathrm{~mL}$ with $\mathrm{RO}$ water

860

861 Diluted NanoLuc Buffer (2x): For plate-based measurement of NanoLuc activity

$8621 \mathrm{~mL}$ Nano-Glo buffer

$863 \mathrm{~mL}$ PBS

$864 \quad 20 \mathrm{~L}$ NanoLuc Substrate (furimazine solution)

865

$8663 \%$ Native Polyacrylamide gels (32 mL, 4 mini gels): For LipoGlo-electrophoresis of ABCLs from larval

867 homogenate

$22.9 \mathrm{~mL}$ RO water

869

$6.4 \mathrm{~mL} 5 \mathrm{x}$ TBE

870

$2.4 \mathrm{~mL}$ 40\% 19:1 polyacrylamide:bis

871

$\rightarrow$ De-gas under vacuum for 30 minutes

$872 \quad 250 \mu \mathrm{L} 10 \%$ APS

873

$20 \mu \mathrm{L}$ TEMED

874

$\rightarrow$ quickly mix by gentle inversion and transfer to casting plates

875

876 Di-I LDL Lipoprotein migration standard: For normalization of electrophoretic mobility in Ladder Units

$877200 \mu \mathrm{L}$ Dil LDL (L3482, Thermofisher Scientific)

$878 \quad 4 \mathrm{~mL} 1 \times \mathrm{TBE}$ 
$.48 \mathrm{~g}$ sucrose (for $10 \%)$

Adjust final volume to $4.8 \mathrm{~mL}$ with TBE

881

$\rightarrow$ Divide into $50 \mu \mathrm{L}$ aliquots and store at $-80^{\circ} \mathrm{C}$

882

883 5x loading dye: For loading homogenate into LipoGlo-electrophoresis gels

884

$4 \mathrm{~g}$ sucrose

885

$25 \mathrm{mg}$ bromophenol blue

886

Adjust to $10 \mathrm{~mL}$ with TBE

887

888

889 Gel imaging solution (1 gel): For in-gel chemiluminescent imaging of NanoLuc

$890 \quad 1 \mathrm{~mL}$ TBE

$891 \quad 2 \mu \mathrm{L}$ furimazine substrate

892

893 Mounting and Imaging solution (1 mL, 20 larvae): For imaging of ABCL distribution in intact larvae

894 .1g low-melt agarose

$895 \quad 10 \mathrm{~mL}$ 1x TBE

896

$\rightarrow$ heat in microwave (5-15 seconds) and swirl until dissolved

897 $\rightarrow$ Distribute to $1 \mathrm{~mL}$ aliquots in $42^{\circ} \mathrm{C}$ heat block

898

Add $10 \mu \mathrm{L}$ furimazine to $1 \mathrm{~mL}$ liquid agarose just prior to mounting

899

900

901 HEPES-Buffered Saline: For establishing density gradient for ultracentrifugation

$902 \quad .85 \mathrm{~g} \mathrm{NaCl}$

$903 \quad 10 \mathrm{~mL} 1 \mathrm{M}$ HEPES buffer $(\mathrm{pH} 7.4)$

$90490 \mathrm{~mL}$ RO water

905 
All datasets were initially subjected to Levene's test for homogeneity of variance. For datasets

910 with a single factor and uniform variance, a one-way ANOVA was used to test for a main effect, and

911 Tukey's HSD was used for post hoc testing. If variance was not uniform (Levene's <.05), Welch's

912 ANOVA with a post hoc Games-Howell test was used as these tests are robust to the assumption of

913 unequal variance. For two-factor datasets, the Robust Two-Factor ANOVA was used with a post hoc

914 Games-Howell test. * denotes $p<.01,{ }^{* *}$ denotes $p<.001$, and ${ }^{* *}$ denotes $p<.0001$. For LipoGlo-

915 electrophoresis experiments, statistical tests were run independently for each of the four groups of

916 binned data (ZM, VLDL, IDL, and LDL). In this case, Bonferroni correction was used to adjust for

917 multiple comparisons (corrected significant $p<.0125$ ). Bonferroni correction was also applied to the

918 LipoGlo-Microscopy experiments which are binned into three groups, so a significant threshold was set

919 at $p<.017$. All statistics were run using XLSTAT, with the exception of the Robust Two-Factor ANOVA

920 which was executed in R using the pbad2way function in the WRS2 package (https://cran.r-

921 project.org/web/packages/WRS2/index.html).

926 KEY RESOURCES TABLE

\begin{tabular}{|l|l|l|}
\hline REAGENT or RESOURCE & SOURCE & IDENTIFIER \\
\hline Critical Commercial Assays & Promega Corp & N1110 \\
\hline Nano-Glo assay & ThermoFisher & L3482 \\
\hline Di-I-Labeled fluorescent LDL & Scientific & \\
\hline Experimental Models: Organisms/Strains & & \\
\hline ApoB-NanoLuc fusion allele (apoBb.1 & & \\
\end{tabular}




\begin{tabular}{|c|c|c|}
\hline Mutant $m t p$ allele $\left(m t p^{s t}\right)$ & Yaniv Lab (Weizmann) & ZIRC stock TBD \\
\hline Mutant apoC2 allele $\left(a p o C 2^{\text {sd38}}\right)$ & Miller Lab (UCSD) & ZIRC stock TBD \\
\hline Mutant pla2g12b allele (pla2g12b $b^{\text {sa659}}$ ) & Sanger Institute & ZIRC stock TBD \\
\hline CNS marker Tg(Xla.Tubb2:mapple-CAAX) & $\begin{array}{l}\text { Halpern Lab } \\
\text { (Carnegie) }\end{array}$ & ZIRC stock TBD \\
\hline \multicolumn{3}{|l|}{ Recombinant DNA } \\
\hline TALEN pair 1 - left arm & $\begin{array}{l}\text { Farber Plasmid Stock } \\
1512\end{array}$ & Addgene stock TBD \\
\hline TALEN pair 1 - right arm & $\begin{array}{l}\text { Farber Plasmid Stock } \\
1513\end{array}$ & Addgene stock TBD \\
\hline TALEN pair 2 - left arm & $\begin{array}{l}\text { Farber Plasmid Stock } \\
1514\end{array}$ & Addgene stock TBD \\
\hline TALEN pair 2 - right arm & $\begin{array}{l}\text { Farber Plasmid Stock } \\
1515\end{array}$ & Addgene stock TBD \\
\hline $\begin{array}{l}\text { Donor plasmid with homology arms flanking NanoLuc } \\
\text { coding sequence }\end{array}$ & $\begin{array}{l}\text { Farber Plasmid Stock } \\
1511\end{array}$ & Addgene stock TBD \\
\hline
\end{tabular}




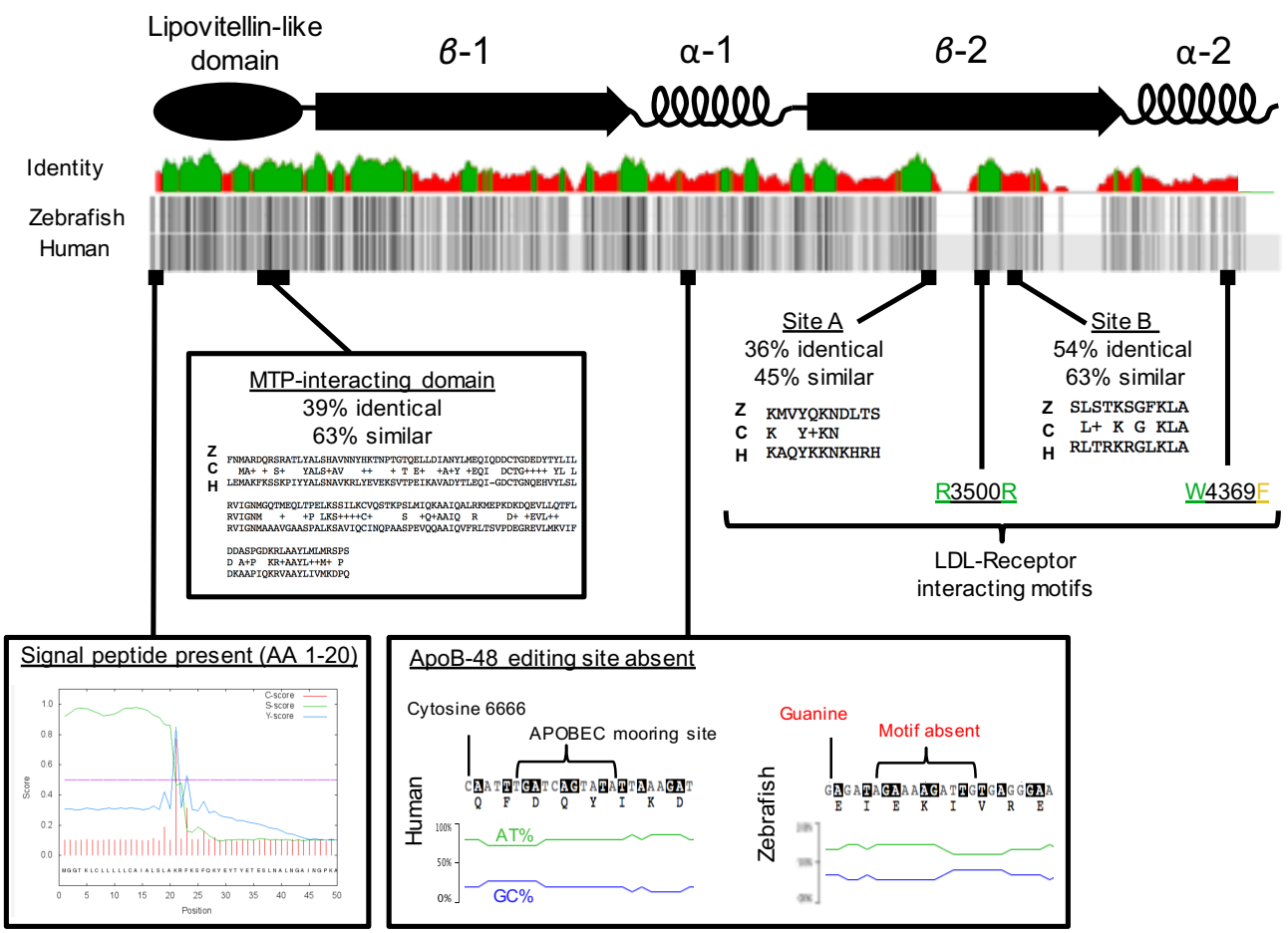

b Coverage

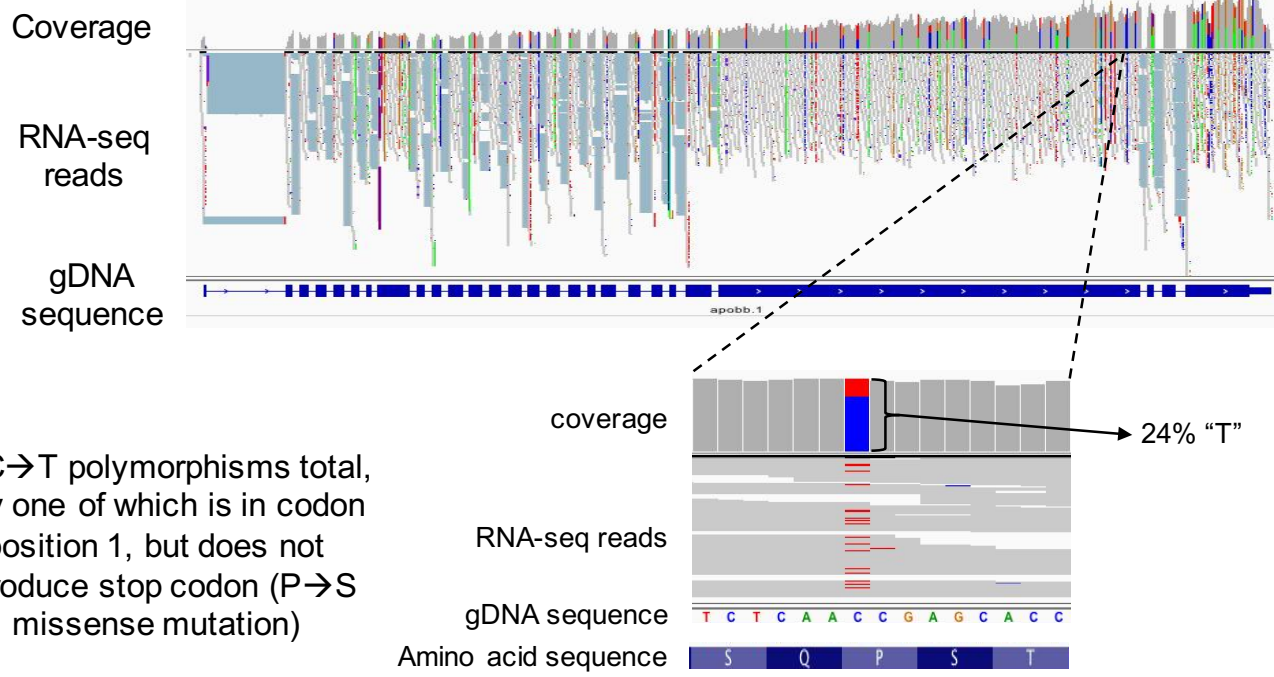

$23 \mathrm{C} \rightarrow \mathrm{T}$ polymorphisms total, only one of which is in codon position 1, but does not introduce stop codon $(P \rightarrow S$ missense mutation)

\section{Supplementary Figure 1:}

Conservation of functional domains in the zebrafish ApoBb.1 ortholog of Human APOB. (a) APOB has a penta-partite domain structure, with an amino-terminal globular domain followed by a series of beta and alpha domains. Consistent with other apolipoprotein sequences, APOB shows relatively low sequence conservation between species at the amino acid level $(25 \%$ identical, $43 \%$ similar, green indicates $>30 \%$ identity in identity plot). However, sequence conservation is enriched in known ApoB functional domains. For example, there is clear conservation of a signal peptide motif at the amino terminus. The MTPinteracting domain shows $39 \%$ identity and $63 \%$ similarity, and the LDL-R interacting motifs are also well-conserved. However, the ApoB-48 editing site appears completely absent, as zebrafish apoBb. 1 lacks the essential C6666 that is edited to form the premature stop, as well as the APOBEC mooring site, and shows only mild AT-richness that has been shown to be important for APOBEC binding (b) To further evaluate whether $a p o B$-editing takes place in zebrafish, RNA reads were mapped back to this genomic locus. Post-transcriptional $C \rightarrow U$ editing would appear as a $C \rightarrow T$ polymorphism in the genomic sequence. 23 instances of $C \rightarrow T$ polymorphism were observed, but the vast majority (21) appeared in the wobble position (position 3 ) of the codon as would be expected for true polymorphisms (rather than post-transcriptional RNA-editing). Of the single instance that occurred in position 1, this did not result in a premature stop codon, providing further support for the absence of APOB-editing activity in zebrafish. 
a

TALEN sites $\frac{\text { Left (TALEN 1) }}{\frac{\text { Left (TALEN 2) }}{. . . T T \text { GAGCTTCCTCTCCCATTTTTCCAGTAATATGGTCATTCTGAACAGAA... }}}$

ApoBb.1 gene

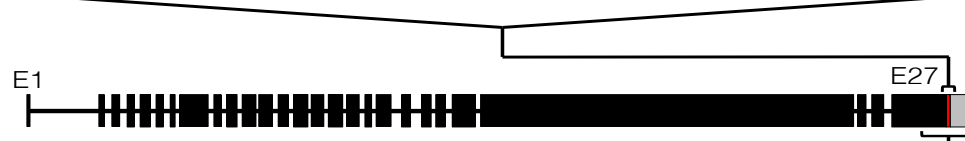

Wild-type locus
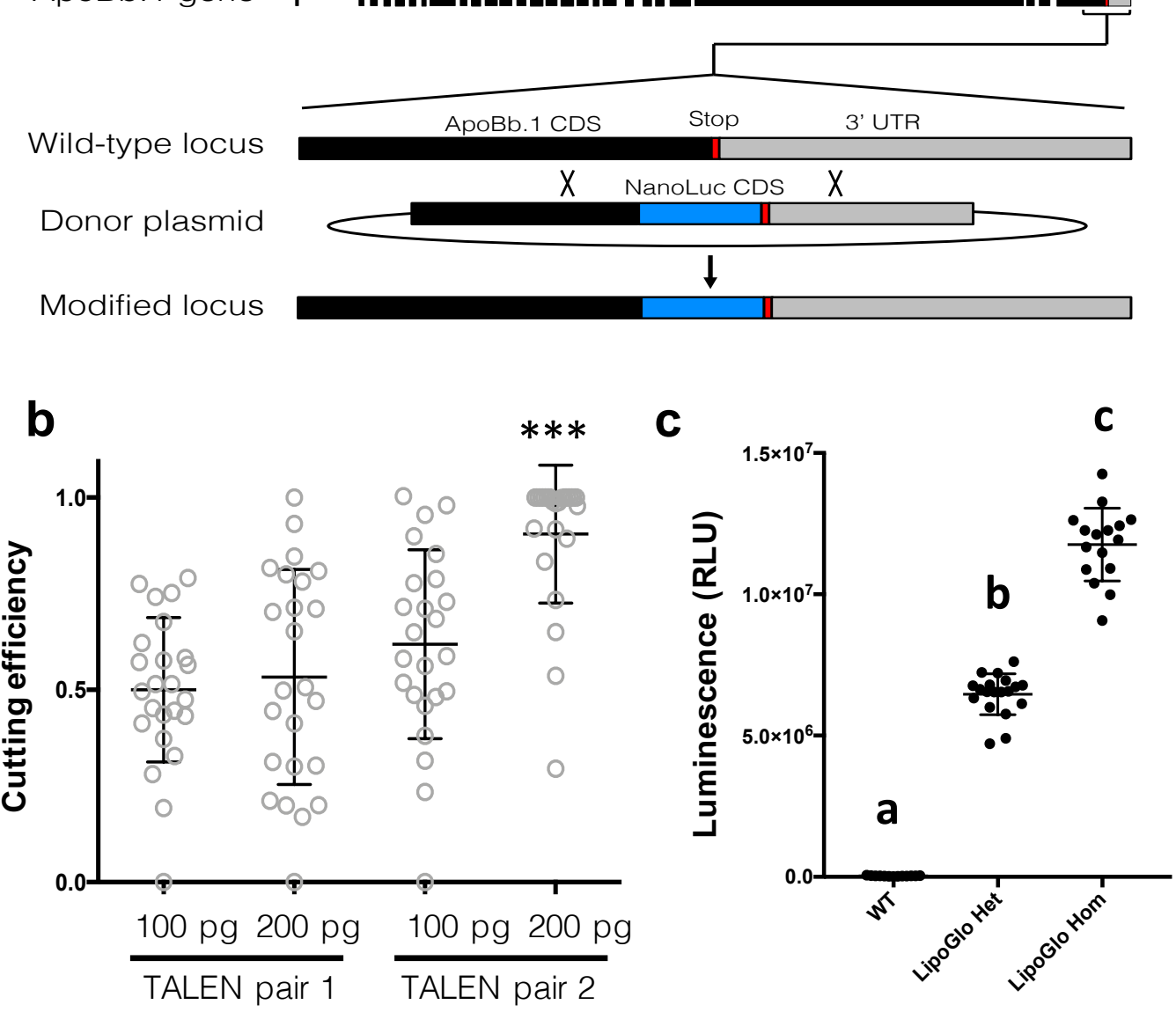

Supplementary Figure 2:

Introduction of an in-frame NanoLuc fusion protein at the endogenous apoBb.1 locus. (a) A Bsrl restriction site overlaps partially with the $a p o B b .1$ stop codon. Two independent pairs of TALENs were designed as shown, and (b) tested for cutting efficiency which was quantified as a loss of susceptibility to Bsrl digest. TALEN pair 2 showed significantly higher cutting efficiency, and was selected for co-injection with the DNA donor construct ( $n=24$, ANOVA p<0.0001, Tukey's HSD p<.0001). (c) An incross of adult fish heterozygous for the LipoGlo reporter revealed the expected mendelian ratio of offspring, and showed that homozygous carriers produce approximately twice the signal intensity as heterozygotes $(1.2 \mathrm{E} 7 \pm 1.3 \mathrm{E} 6 \mathrm{vs} 6.5 \mathrm{E} 6 \pm 7.3 \mathrm{E} 5)$ $(n \approx 16$, ANOVA $p<0.0001$, Tukey's HSD $p<.0001$ ). Heterozygous and homozygous carriers of the LipoGlo reporter are viable, fertile, and free of overt morphological defects. 
a

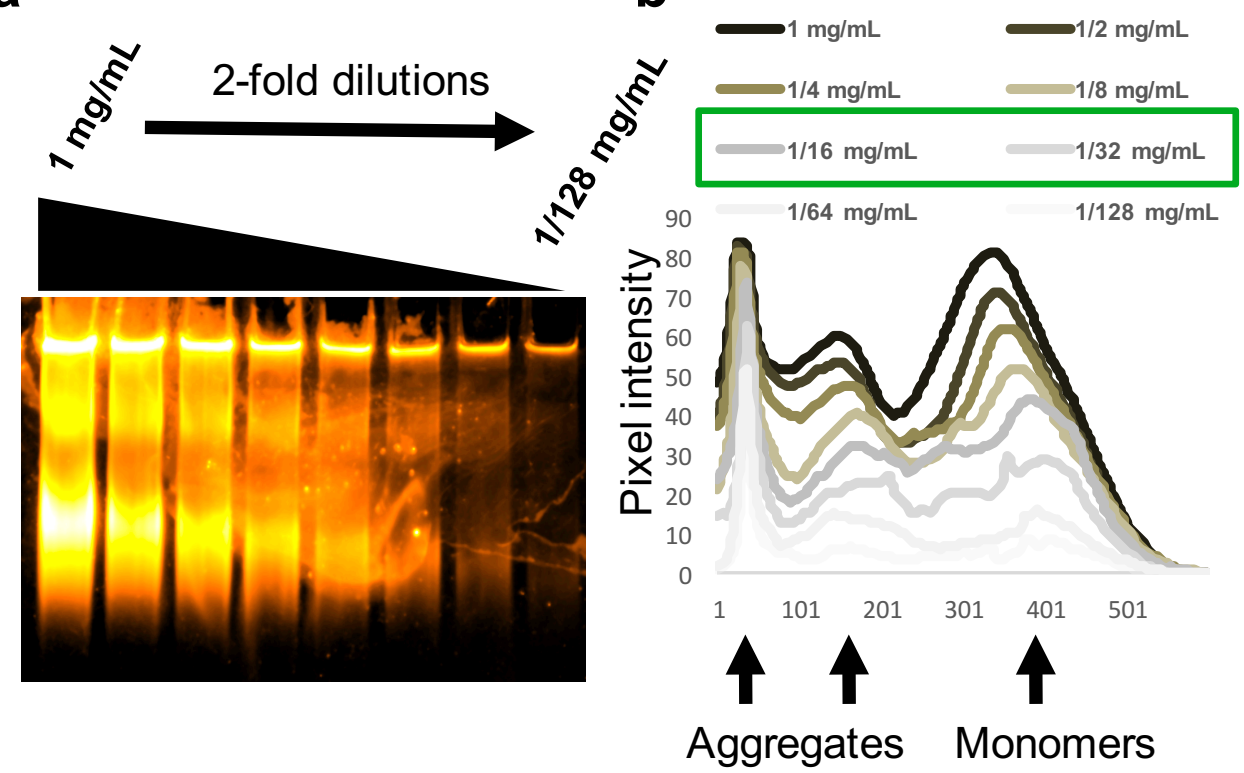

C \# of freeze-thaw cycles:

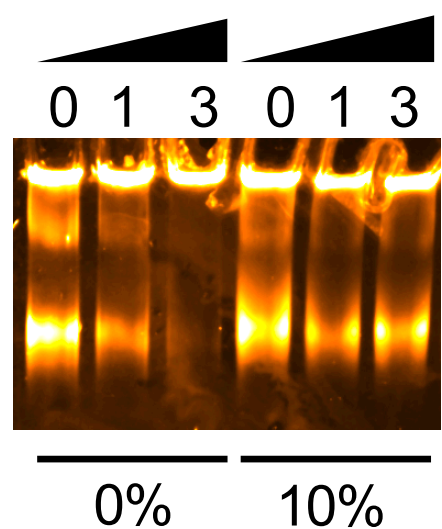

Percent sucrose (cryoprotectant) d

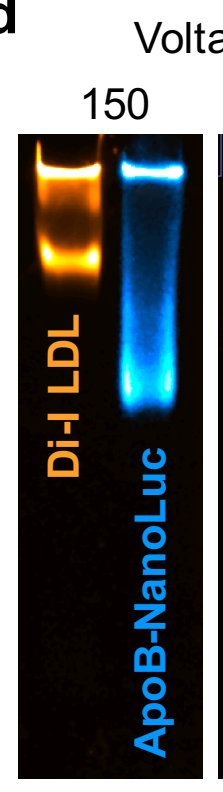

e

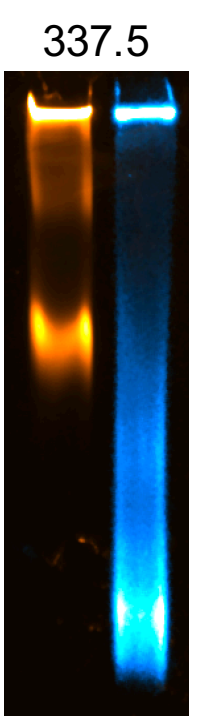

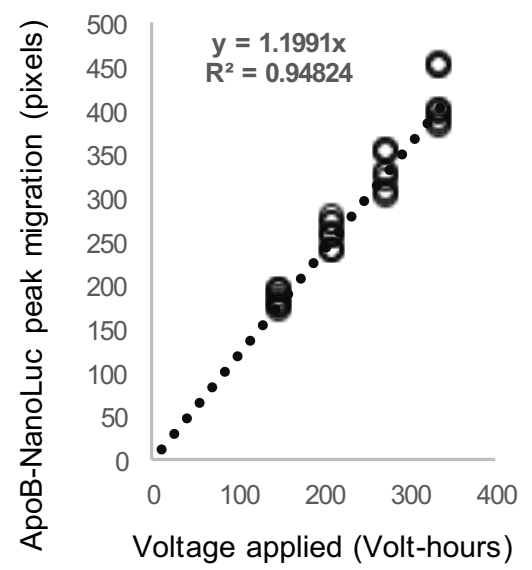

Coefficient of Variation: $6.1 \%$ f

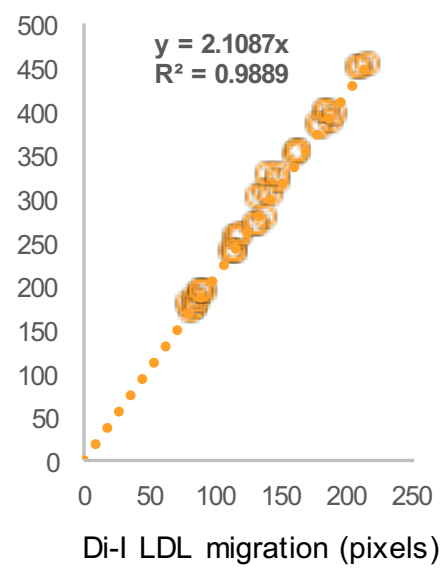

Coefficient of Variation: $3.2 \%$

\section{Supplementary Figure 3:}

Development of an effective migration standard for lipoprotein gels. It is essential that lipoprotein gels include a ladder or normalization standard that has similar electrophoretic properties to ABCLs. Di-I labeled human LDL serves as a commercially available option that enables standardization not only between multiple gels but also between different labs. (a) Di-I LDL was subjected to a series of 2-fold dilutions and separated via Native-PAGE as described and imaged with the Licor-Fc to determine an appropriate dilution factor that was still readily detectable. (b) Plot profiles of each of the serial dilutions revealed retardation of peak mobility in the highly concentrated samples, potentially due to overcrowding. Dilution factors between 16 and 32-fold were selected as acceptable (green box), and a 24-fold dilution was used for subsequent assays. (c) Sucrose was included as a cryoprotectant during Di-I LDL dilution, and there is no change in peak particle mobility across at least 3 freezethaw cycles in the presence of $10 \%$ sucrose, whereas the ladder is almost completely aggregated without cryoprotectant. (d) To determine the relationship between mobility of the standard and lipoprotein samples, homogenate was prepared and pooled from $\mathrm{mtp}$-/- (3 dpf) mutant larvae (which produce primarily LDL-like particles). Samples of homogenate were run alongside Di-I standard for either 150, 212.5, 275, or 337.5 volt-hours, and the peak migration (in pixels) was quantified for each species. (e) While there was a clear linear relationship between $A B C L$ migration and voltage applied $\left(R^{2}=.95\right)$, (f) the relationship was much tighter when electrophoretic mobility was compared to the migration standard $\left(R^{2}=.99\right)$, validating the utility of $\mathrm{Di}-\mathrm{I} L \mathrm{~L} L$ as a migration standard. 

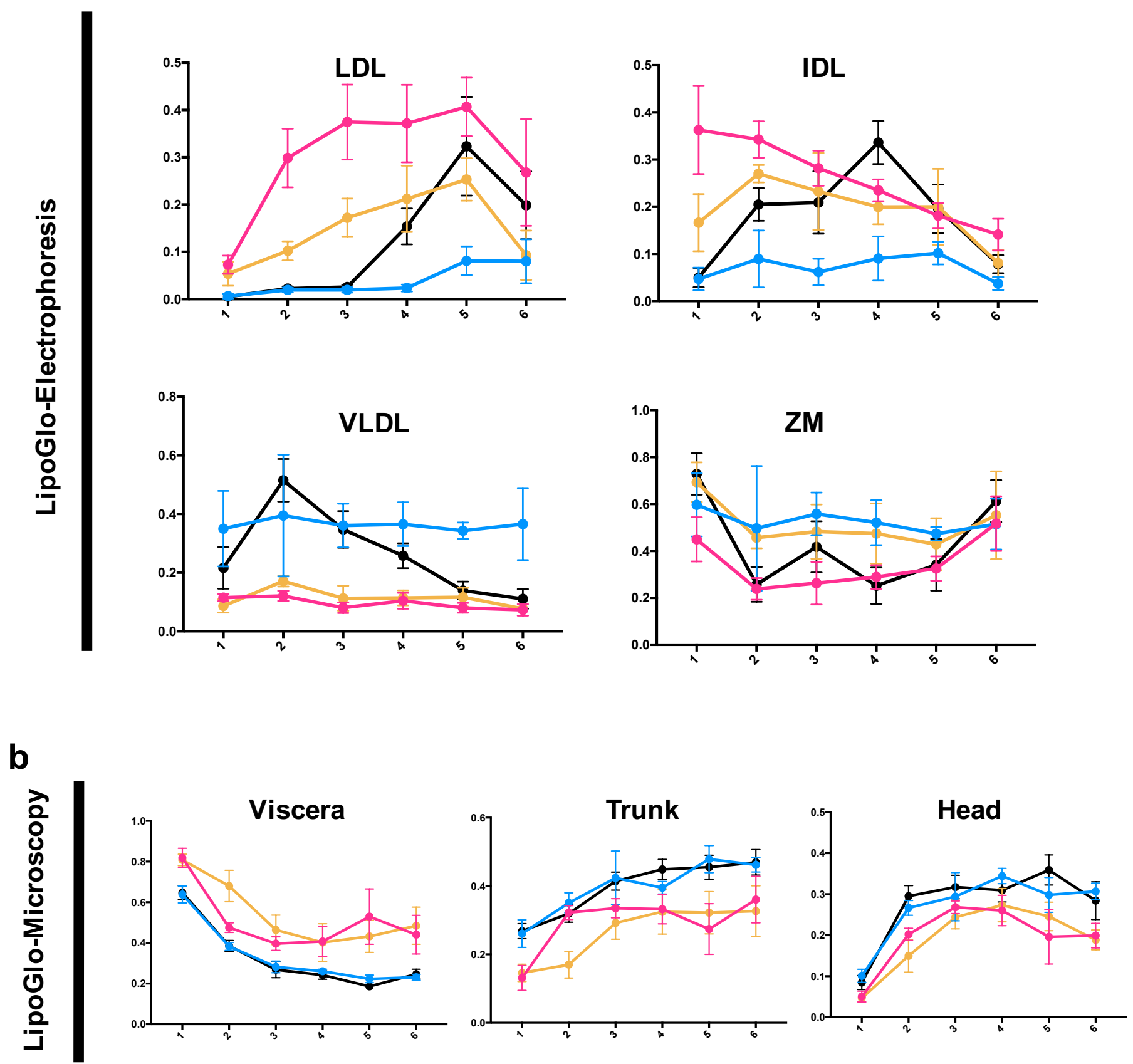

Supplementary Figure 4:

Side-by-side analysis of LipoGlo-Electrophoresis and Microscopy results from mutant genotypes. (a) Plots of electrophoresis and (b) microscopy data reported in the main text grouped by subclass rather than by genotype and showing standard deviations. 
a

Brightfiled + Tg(Xla. Tubb2-mApple-CAAX)

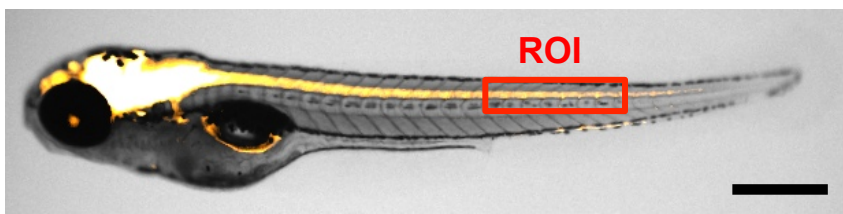

$\operatorname{Tg}($ Xla. Tubb2-

mApple-CAAX)

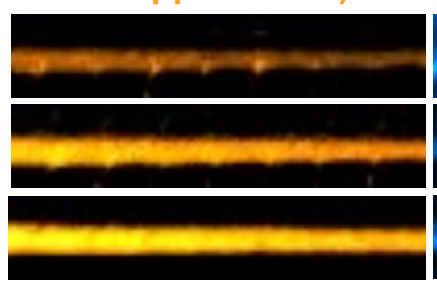

ApoB-NanoLuc

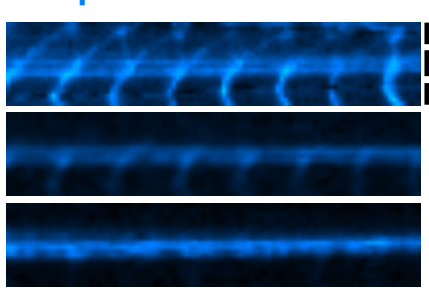

b

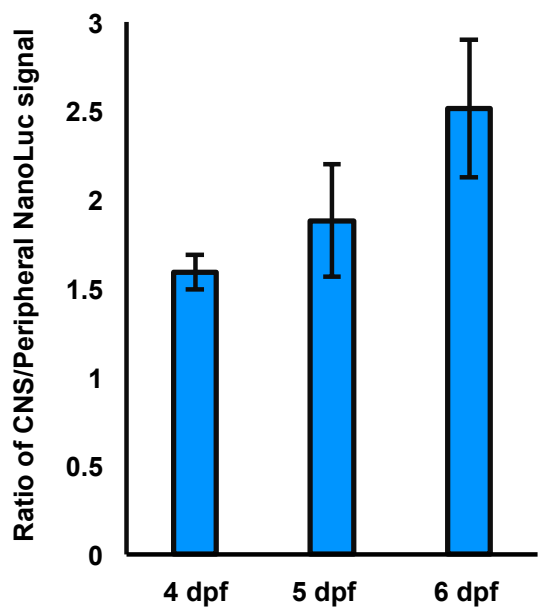

C
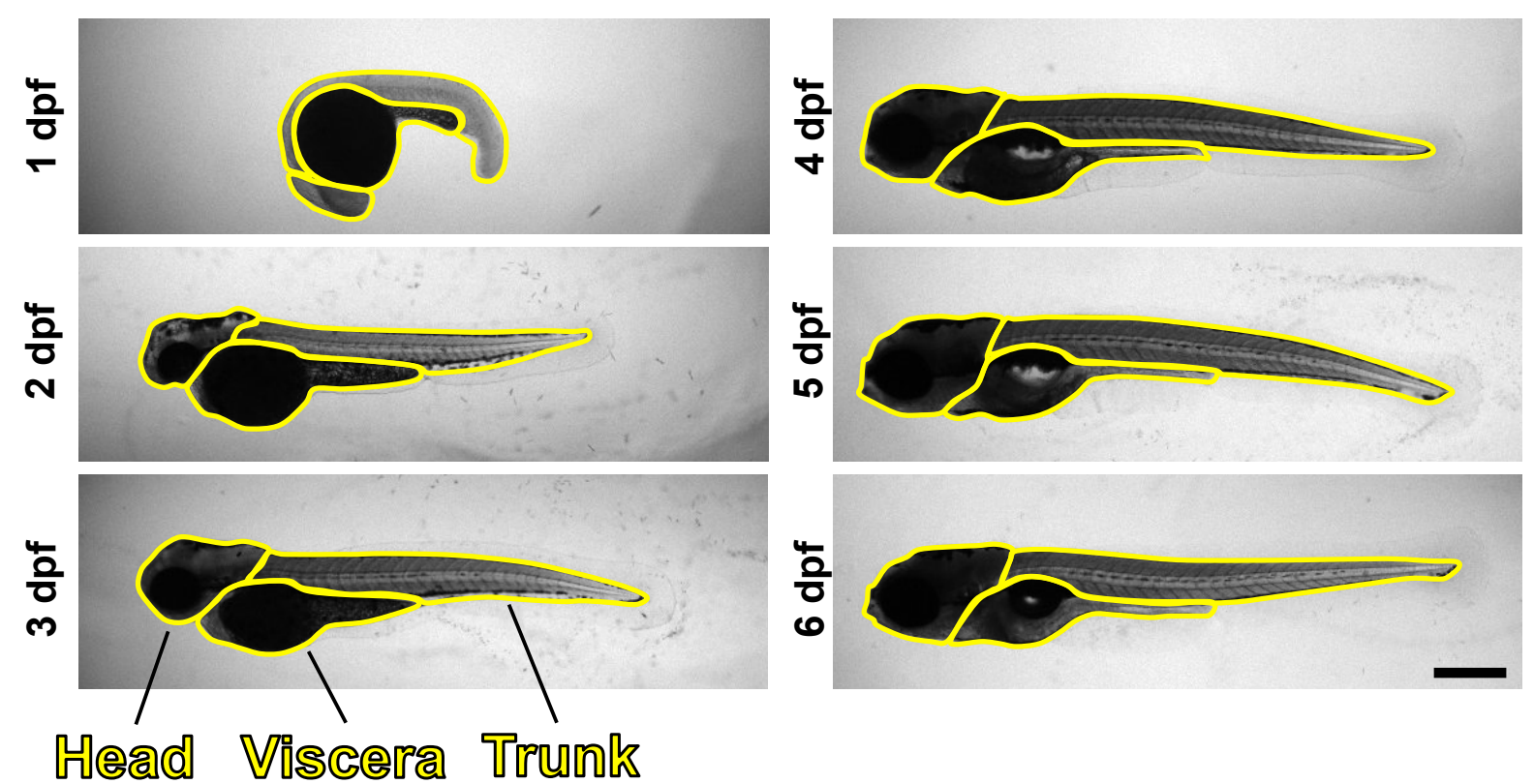

Supplementary Figure 5:

LipoGlo microscopy reveals ABCL localization. (a) Three independent clutches of larvae carrying both the CNS marker $T g(X / a$. Tubb2-mApple-CAAX) and ApoB-NanoLuc fusion were fixed and imaged at 4, 5, and $6 \mathrm{dpf}$ as described in the methods section. A 20x100 pixel region of interest (ROI) was drawn centered around the spinal cord (marked by mApple) just distal to the intestine. The mApple and ApoB-NanoLuc channels are displayed separately below (representative of 15 images per time point). (b) Quantification of the signal intensity in spinal cord (CNS) versus peripheral regions revealed a gradual enrichment of signal in the CNS relative to the periphery from 4-6 dpf ( $n=15$, Welch's ANOVA $p<0.0001$, Games-Howell $p<.01)$. (c)

Representative images of regions of interest corresponding to viscera, trunk, and head regions across development. Scale bars $=500 \mu \mathrm{m}$. 
a

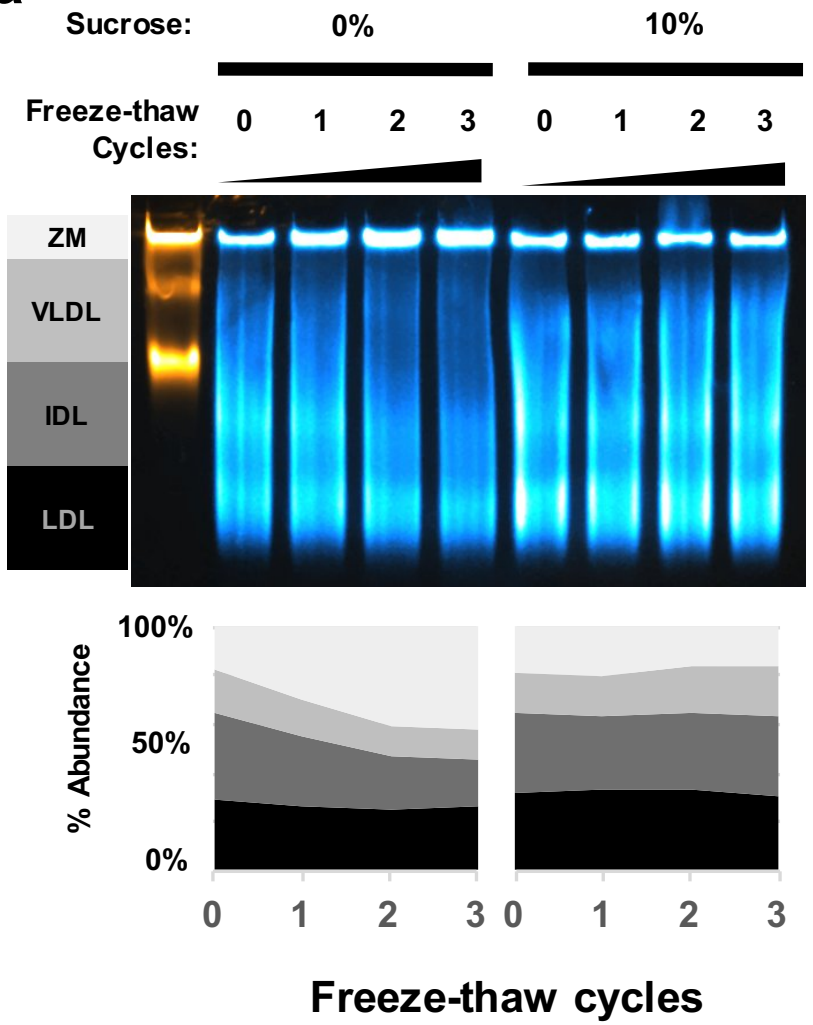

b

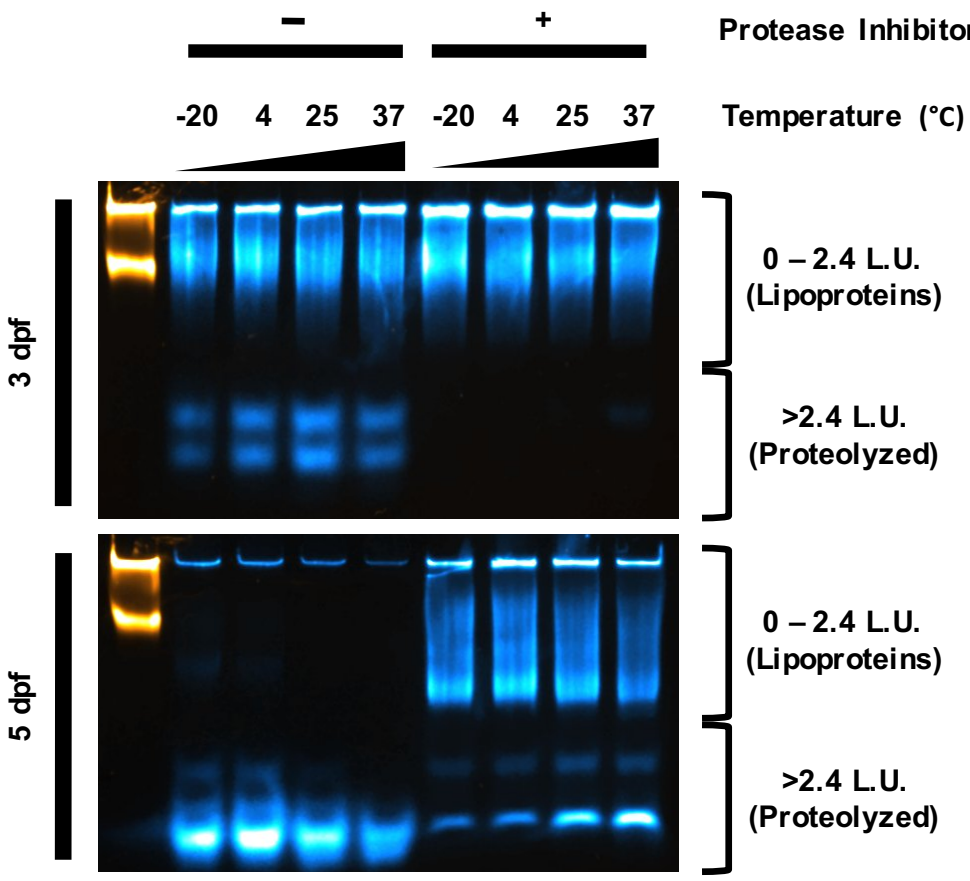

Supplementary Figure 6:

Cryoprotectant and protease-inhibition properties of $A B C L$ stabilization buffer. (a) $4 \mathrm{dpf}$ wild-type larvae were homogenized in $\mathrm{ABCL}$ stabilization buffer containing $0 \%$ or $10 \%$ final concentration of sucrose and subjected to between 0 and 3 freeze-thaw cycles, and then separated using LipoGlo-electrophoresis as described in the methods section. While the lipoprotein size distribution remained constant in samples containing sucrose as a cryoprotectant, samples without sucrose showed a gradual enrichment of ZM particles, which appears to be due to aggregation of VLDL and IDL particles. (b) Larvae were homogenized in $A B C L$ stabilization buffer with and without the protease inhibitor components (cOmplete mini EDTA-free tablet supplemented with $40 \mathrm{mM}$ final concentration of EGTA, see recipes) and incubated at various temperatures for 2 hours. Samples were then separated by LipoGlo-electrophoresis at $50 \mathrm{~V}$ for 30 minutes, and $125 \mathrm{~V}$ for 60 minutes. This is $125 \mathrm{Volt}$-hours less than described in the methods section to enable visualization of proteolysis products. At $3 \mathrm{dpf}$, protease activity is quite low such that no proteolyzed products are present in the group treated with protease inhibitor, whereas degradation products are visible in a temperature-dependent manner in the absence of inhibitors. By $5 \mathrm{dpf}$, protease activity is much higher in the homogenate sample, presumably due to development of a mature intestine. Protease activity is still well-controlled in the presence of protease inhibitor at low temperatures, but in the absence of protease inhibitor degradation is so severe that there are signs of both cleavage of NanoLuc from the lipoprotein particle as well as proteolysis of the reporter itself. 
a

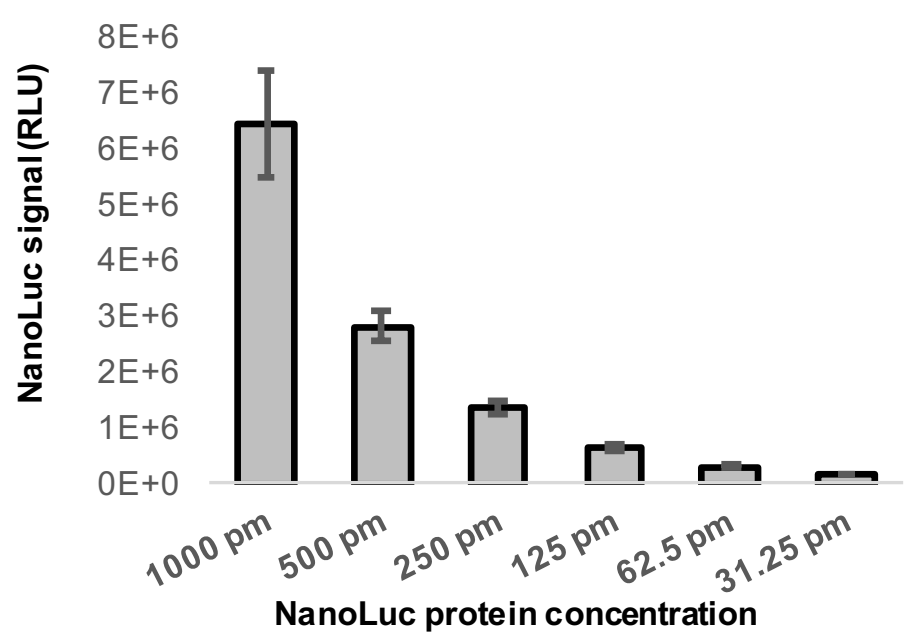

C

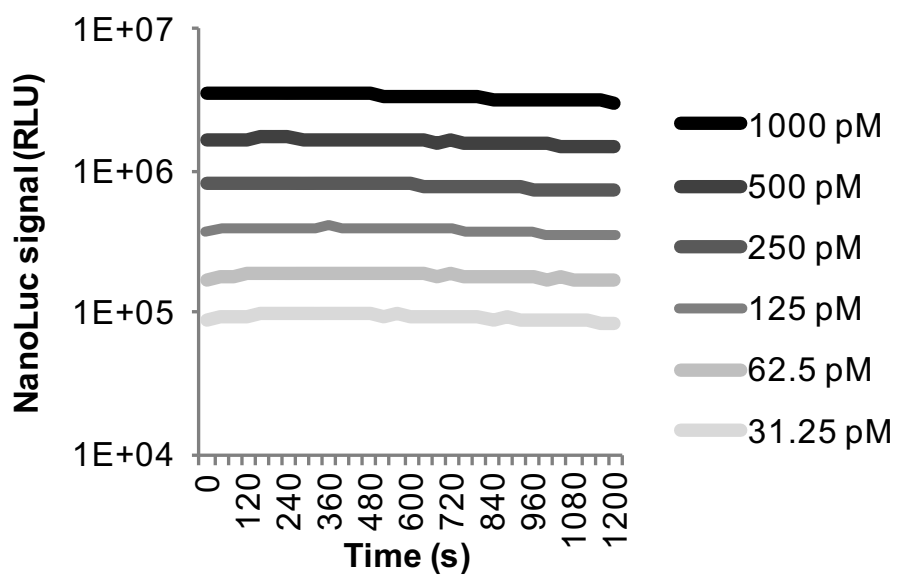

b

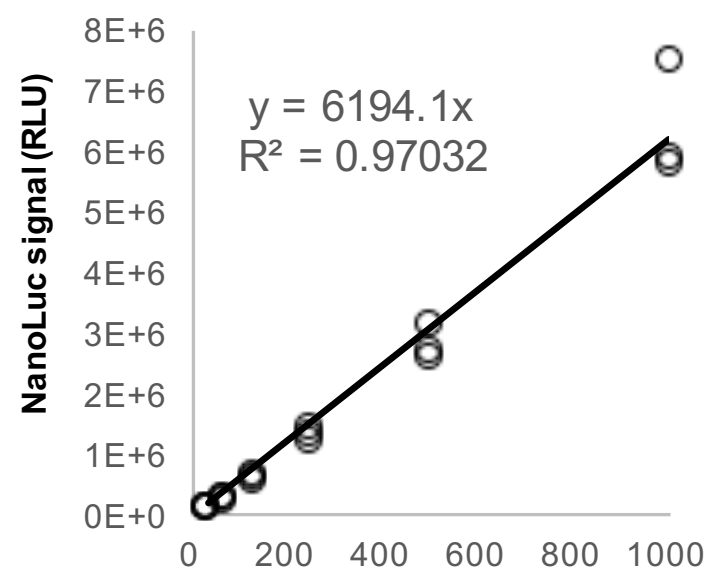

NanoLuc protein concentration (picomolar) d

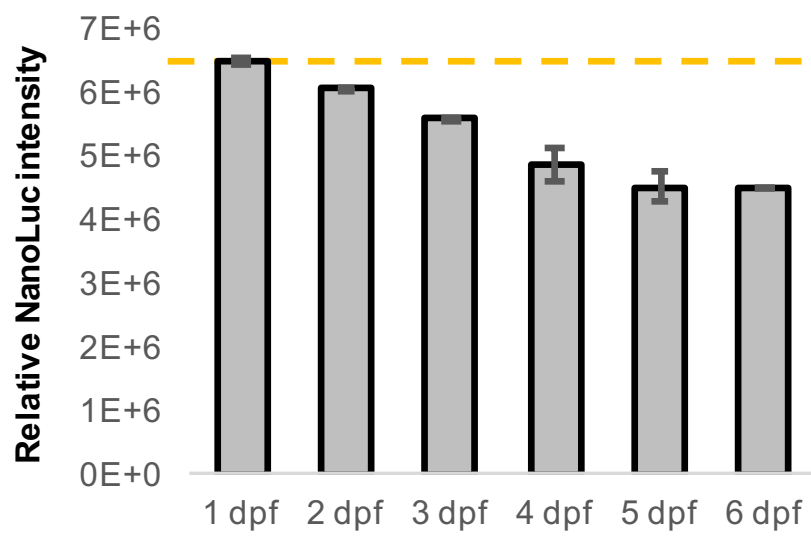

Developmental stage of larval homogenate

\section{Supplementary Figure 7:}

NanoLuc standard curves. (a) To determine the absolute concentration of ABCLs in the larval homogenate, purified NanoLuc protein was ordered directly from Promega (Nluc-HT Protein, 500ug, 54.2KDa, \#CS188401) and diluted to $1 \mathrm{nM}$ working concentration in $1 \times A B C L$ stabilization buffer. This solution was subjected to a 6-point series of 2-fold dilutions and used in a plate-based assay for NanoLuc activity, and (b) luminescent signal showed excellent linear correlation with protein concentration within this concentration range $\left(\mathrm{R}^{2}=.97\right)$. (c) Plate reads were repeated in a kinetic experiment reading well values every 40 seconds for 20 minutes. Signal decayed only marginally in this time window, and half-lives were calculated to be greater than 60 minutes for all concentrations tested. (d) There is a marked increase in pigmentation throughout larval development, causing homogenate to become progressively more opaque. To test the effect of pigment on NanoLuc readings, wild-type larvae that lack the ApoB-NanoLuc reporter were homogenized in ABCL stabilization buffer at each day of larval development. This homogenate was then supplemented with a final concentration of $1 \mathrm{nM}$ NanoLuc protein and subjected to a plate read assay. As expected, the relative intensity of NanoLuc signal declines from 1-6 dpf, indicating that absolute quantitation of NanoLuc levels should include a standard curve that accounts for changes in larval pigmentation. 


\begin{tabular}{|c|l|l|}
\hline Primer \# & \multicolumn{1}{|c|}{ Purpose } & \multicolumn{1}{c|}{ Sequence } \\
\hline 1 & generate pME NanoLuc $F$ & GGG GAC AAG TTT GTA CAA AAA AGC AGG CTT GAT GGT CTT CAC ACT CGA AGA TTT C \\
\hline 2 & generate pME NanoLuc $R$ & GGG GAC CAC TTT GTA CAA GAA AGC TGG GTT TAC GCC AGA ATG CGT TCG CA \\
\hline 3 & generate left homology arm $F$ & GGG GAC AAC TTT GTA TAG AAA AGT TGC GCT GCC TGG AAT GAA TGA AGC \\
\hline 4 & generate left homology arm $R$ & GGG GAC TGC TTT TTT GTA CAA ACT TGT CTG GAA AAA TGG GAG AGG AAG \\
\hline 5 & generate right homology arm F & GGG GAC AGC TTT CTT GTA CAA AGT GGA ATA TGG TCA TTC TGA ACA GAA AGT AAA \\
\hline 6 & generate right homology arm R & GGG GAC AAC TTT GTA TAA TAA AGT TGG GTA AGG CAG ACA TCA GTT TGT AAG \\
\hline 7 & test TALEN cutting efficiency $F$ & TGC AAT GAA GCA AAT CGA AAG TC \\
\hline 8 & test TALEN cutting efficiency $R$ & AAG ATT GGG TCG TGT TGC AT \\
\hline 9 & genotype LipoGlo $F$ & GCT TCC TCT CCC ATT TTT CC \\
\hline 10 & genotype LipoGlo R1 & CCC CGA GAT TCT GAA ACA AAC \\
\hline 11 & genotype LipoGlo R2 & AAG TGT CCA TTG GCT TCG AT \\
\hline 12 & genotype mtp $F$ & GTC TGA GGT TCA GAT GTA CCT GTT AGG AC \\
\hline 13 & genotype mtp R & CTC TGC TGT GAT GAG CGC AGG \\
\hline 14 & genotype apoc2 F & GAG CGG AGA GCT TTC GTG T \\
\hline 15 & genotype apoc2 $R$ & CTT CCA GCT TGT AGC CCT TG \\
\hline 16 & genotype pla2g12b $\mathrm{F}$ & ACA AGG GAA AGC AAA CCA AA \\
\hline 17 & genotype pla2g12b $R$ & CAG TGT TGT ACA TGG TGT CTG C \\
\hline & &
\end{tabular}

\section{Supplementary Table 1:}

\section{Primers used in this study.}

